\title{
The integrin beta 1 modulator Tirofiban prevents adipogenesis and obesity by the overexpression of integrin-linked kinase: a pre-clinical approach in vitro and in vivo
}

S. de Frutos ${ }^{1,2,3,4^{*}}$ (D, M. Griera ${ }^{1,2,3}$, M. Hatem-Vaquero ${ }^{1,2}$, S. Campillo ${ }^{1,2}$, E. Gutiérrez-Calabres ${ }^{1,2}$, D. García-Ayuso ${ }^{1,2}$, M. Pardo ${ }^{5}$, L. Calleros ${ }^{1,2}$, M. Rodríguez-Puyol ${ }^{1,2}$ and D. Rodríguez-Puyo ${ }^{2,4,6}$

\begin{abstract}
Background: Obesity is caused by the enlargement of the white adipose tissue (WAT) depots, characterized by the hypertrophic enlargement of malfunctioning adipocytes within WAT which increases the storage of triglycerides (TG) in the lipid droplets (LD). Adipogenesis pathways as well as the expression and activity of some extracellular matrix receptors integrins are upregulated. Integrin $\beta 1$ (INTB1) is the main isoform involved in WAT remodeling during obesity and insulin resistance-related diseases. We recently described Integrin Linked Kinase (ILK), a scaffold protein recruited by INTB1, as an important mediator of WAT remodeling and insulin resistance. As the few approved drugs to fight obesity have brought long-term cardiovascular side effects and given that the consideration of INTB1 and/or ILK modulation as anti-obesogenic strategies remains unexplored, we aimed to evaluate the anti-obesogenic capacity of the clinically approved anticoagulant Tirofiban (TF), stated in preclinical studies as a cardiovascular protector.
\end{abstract}

Methods: Fully differentiated adipocytes originating from $\mathrm{C} 3 \mathrm{H} 10 \mathrm{~T} 1 / 2$ were exposed to TF and were co-treated with specific INTB1 blockers or with siRNA-based knockdown ILK expression. Lipid-specific dyes were used to determine the TG content in LD. The genetic expression pattern of ILK, pro-inflammatory cytokines (MCP1, IL6), adipogenesis (PPARY, Leptin), thermogenesis (UCP1), proliferation (PCNA), lipid metabolism (FASN, HSL, ATGL), and metabolite transporters (FABP4, FAT, AQP7) were detected using quantitative PCR. Cytoskeletal actin polymerization was detected by confocal microscopy. Immunoblotting was performed to detect INTB1 phosphorylation at Thr788/9 and ILK activity as phosphorylation levels of protein kinase B (AKT) in Ser473 and glycogen synthase kinase 3 $\beta$ (GSK3 $\beta$ ) at Ser9. TF was intraperitoneally administered once per day to wildtype and ILK knockdown mice (CKDILK) challenged with a high-fat diet (HFD) or control diet (STD) for 2 weeks. Body and WAT weight gains were compared. The expression of ILK and other markers was determined in the visceral epididymal (epi) and inguinal subcutaneous (sc) WAT.

Results: TF reduced TG content and the expression of adipogenesis markers and transporters in adipocytes, while UCP-1 expression was increased and the expression of lipases, cytokines or PCNA was not affected. Mechanistically, TF rapidly increased and faded the intracellular phosphorylation of INTB1 but not AKT or GSK3 $\beta$. F-actin levels were rapidly decreased, and INTB1 blockade avoided the TF effect. After $24 \mathrm{~h}$, ILK expression and phosphorylation rates of

\footnotetext{
*Correspondence: sergio.frutos@uah.es

${ }^{1}$ Departamento de Biología de Sistemas, Unidad Fisiología, Facultad de

Medicina, Universidad de Alcalá, A2 KM 33,600, Campus, 28805 Alcalá de Henares (Madrid), Spain

Full list of author information is available at the end of the article
} original author(s) and the source, provide a link to the Creative Commons licence, and indicate if changes were made. The images or other third party material in this article are included in the article's Creative Commons licence, unless indicated otherwise in a credit line to the material. If material is not included in the article's Creative Commons licence and your intended use is not permitted by statutory regulation or exceeds the permitted use, you will need to obtain permission directly from the copyright holder. To view a copy of this licence, visit http://creativecommons.org/licenses/by/4.0/. The Creative Commons Public Domain Dedication waiver (http://creativeco mmons.org/publicdomain/zero/1.0/) applies to the data made available in this article, unless otherwise stated in a credit line to the data. 
AKT and GSK3 $\beta$ were upregulated, while ILK silencing increased TG content. INTB1 blockade and ILK silencing avoided TF effects on the TG content and the transcriptional expression of PPARY and UCP1. In HFD-challenged mice, the systemic administration of TF for several days reduced the weight gain on WAT depots. TF reduced adipogenesis and pro-inflammatory biomarkers and increased lipolysis markers HSL and FAT in epiWAT from HFD, while increased UCP1 in scWAT. In both WATS, TF upregulated ILK expression and activity, while no changes were observed in other tissues. In HFD-fed cKDILK, the blunted ILK in epiWAT worsened weight gain and avoided the anti-obesogenic effect of in vivo TF administration.

Conclusions: ILK downregulation in WAT can be considered a biomarker of obesity establishment. Via an INTB1-ILK axis, TF restores malfunctioning hypertrophied WAT by changing the expression of adipocyte-related genes, increasing ILK expression and activity, and reducing TG storage. TF prevents obesity, a property to be added to its anticoagulant and cardiovascular protective advantages.

Keywords: Tirofiban, Integrin-beta1, Integrin-linked kinase, AKT, Adipogenesis, Obesity, Hypertrophic adipocyte, Browning, White adipose tissue, Lipolysis, Inflammation

\section{Background}

Obesity is a chronic disease related to white adipose tissue (WAT) that increases the risk of metabolic disorders such as diabetes mellitus type 2, non-alcoholic fatty liver disease, atherosclerosis, and arterial damage. The dysfunctional AT is unable to efficiently process the circulating metabolites and hyperlipidemia and/or hyperglycemia lead other organs to malfunction [1]. Under physiological conditions the adipocytes properly manage the uptake and disposal of triglycerides (TG) through lipogenic and lipolytic mechanisms. AT function is sustained by the continuous adipogenesis or the differentiation of pre-adipocytes to adipocytes [2]. During obesity, WAT depots enlarge by either the increase of the adipocyte number, known as WAT hyperplasia, or the shape rearrangement of the volume of pre-existing adipocytes by the increasing TG formation (lipogenesis) and storage in lipid droplets (LD), known as hypertrophy. The adipocytes' differentiation state can be tracked by the expression and activity of biomarkers, such as the proinflammatory cytokines Monocyte Chemoattractant Protein-1 (MCP1) and Interleukin-6 (IL6); adipogenesis and differentiation genes, such as Peroxisome ProliferatorActivated Receptor-gamma (PPAR $\gamma$ ) and the adipokine Leptin; beige/brown adipocyte marker Uncoupling Protein-1 (UCP1); proliferating cell nuclear antigen (PCNA); lipid metabolism enzymes, such as Fatty Acid Synthase (FASN), Hormone Sensitive Lipase (HSL) and Adipose Triglyceride Lipase (ATGL); and TG metabolite transporters, such as Fatty Acid Binding Protein 4 (FABP4), Fatty Acid Translocase (FAT) and Aquaglyceroporin 7 (AQP7). The expression of these biomarkers by the adipocytes, as well as the quantity and quality of extracellular matrix $(E C M)$ surrounding them are changed in the malfunctioning WAT, [3-14]. To orchestrate ECM components with downstream intracellular architecture, all cells have adhesion receptors called integrins
(INT), which are transmembrane heterodimers consisting of two subunits $\alpha$ and $\beta$ [15]. INT subunits have been involved in obesity and insulin resistance $[16,17]$, particularly Integrin $\beta 1$ (INTB1), which is the main WAT subunit and has been profusely studied as a final determinant of adipocyte differentiation [18-21]. INT1B activity is controlled by conformational structure switches and a bidirectional outside-in and inside-out signaling. INTB1 inside-out activation is initiated through the specific phosphorylation in T788/T789 of the cytoplasmic domain by components of the intracellular protein cluster, known as adhesome or interactome, and a requisite to conformationally change INTB1 from low-affinity to high-affinity binding with the ECM substrate and the facilitation of the outside-in signaling [22]. During the development of obesity, erratic ECM-INTB1 interaction causes WAT to malfunction through the remodelling of the adipocyte's intracellular skeleton, which changes in LDs configuration and metabolites trafficking processes [23-27]. However, few publications have noted that ECM-based molecules may be used as a therapeutic strategy $[5,9,17]$. INTB1 lacks kinase activity, but several downstream intracellular kinases recruited in the interactome may be involved during the communication process, although the hierarchy and relevancy of each is not completely defined. Some of these kinases are protein kinase $\mathrm{B}$ (also known as AKT), glycogen synthase kinase $3 \beta$ (GSK3 $\beta$ ) or focal adhesion kinase (FAK) [28-31]. A major scaffold protein of the interactome recruited during INTB1 modulations is Integrin Linked Kinase (ILK) [32]. The role of ILK in obesity is relatively understudied. Some publications have timidly highlighted changes in ILK during adipogenesis in stem cells [33-35]. Few recent publications, including ours, have described ILK as a mediator during insulin-resistance and WAT remodeling in cells and mice models [36-38]. Most INT-target drugs are based on the amino acid consensus motif 
Arg-Gly-Asp (RGD), featured in ECM proteins and disintegrins from snakes venom [39]. Some competitively block fibrinogen binding to the INTs which are present in the platelets, and thus they are clinically used as an anticoagulant in patients with acute coronary syndromes undergoing percutaneous coronary intervention. The non-peptidic INT blocker Tirofiban (TF, Aggrastat) was the first molecule used for this purpose. Other INTblockers are peptide-based, such as Eptifibatide (EPT), the clinical-discontinued Cilengitide (CIL) or Arg-GlyAsp-Ser (RGDS) [39, 40]. The study of the functionality of INT-targeted drugs on other types of cells apart from platelets is promising [41]. For example, some preclinical studies, including ours, found a wider cardiovascular protective use for TF, rather than its intrinsic anticoagulant properties. [42-50]. To our knowledge, a pharmacological anti-obesogenic approach has not been performed previously with any clinically approved INTB1-targeted drug. Here, we performed experimental approaches in cultured differentiated adipocytes, originating from C3H10T1/2 stem cells [51], with or without a pharmacological INTB1 blockade or the transgenic depletion of ILK. TF was administered systemically in wildtype (WT) and ILK knockdown (cKDILK) mice during the establishment of obesity based on a high-fat diet (HFD) [36]. We determined body and WAT weight gains, ILK expression and activity, and adipogenesis and lipid metabolism markers, as discerned from the cultured cell model. We demonstrated the use of TF as anti-obesogenic and defined the relevance of ILK in the process.

\section{Methods}

\section{Adipocytes culture and differentiation}

C3H10T1/2 preadipocytes (CCL-226, ATCC, Manassas, USA) [43] were grown on 6-well plates with DMEM (Sigma-Aldrich, St. Louis, MO, USA) and 10\% fetal bovine serum (FBS, Thermo Fisher Scientific Waltham, MA, USA). 2 days after cells reached confluency, they were induced to differentiate with DMEM supplemented with 10\% FBS and Glutamax (Thermo Fisher Scientific), $1 \mu \mathrm{M}$ dexamethasone, $1 \mu \mathrm{M}$ rosiglitazone, $0.5 \mathrm{mM}$ 3-isobutyl-1-methylxanthine (IBMX, Sigma-Aldrich) and $5 \mu \mathrm{g} / \mathrm{ml}$ insulin (Merck, Sigma-Aldrich) for $48 \mathrm{~h}$. Medium was replaced every two days for a total of 6-10 days with DMEM supplemented with Glutamax, 10\% FBS and $5 \mu \mathrm{g} / \mathrm{ml}$ insulin. The 9 th days from the beginning of the differentiation, adipocytes were deprived of supplementation for $24 \mathrm{~h}$. C2C12 myoblasts (CRL-1772, ATCC) and monocytes line THP-1 (TIB-202, ATCC) were grown in DMEM or RPMI (Sigma-Aldrich), respectively, with $10 \%$ FBS. Treatments were added under deprived conditions for the indicated times.

\section{Adipocytes transient transfection with siRNAs}

siRNAs transfection was achieved in adhered adipocytes as described [52, 53]. Briefly, fully differentiated adipocytes were plated at $70-80 \%$ confluence in 6 well plates. $24 \mathrm{~h}$ later, adhered adipocytes were transfected with $20 \mathrm{nM}$ specific siRNAs against ILK (silLK, Santa Cruz Biotechnologies Inc., Dallas, TX) or scramble siRNAs with Metafectene (Biontex, Munich, Germany) overnight and allowed to recover with DMEM $+10 \%$ FBS for another $24 \mathrm{~h}$. Then, cells were deprived for $24 \mathrm{~h}$ and treated for the indicated times.

\section{LD staining and TG quantification}

Fully differentiated adipocytes were deprived, treated, washed, and stained after the indicated times with lipid dyes. AdipoRed (Lonza, Basel, Switzerland) was used as indicated by the commercial manual. Fluorimetry was quantified using a plate reader with $485 \mathrm{~nm}$ excitation/572 nm emission (VictorX4 from Perkin-Elmer) and values were normalized to total protein content per well, determined by DC-Protein Assay (Bio-Rad, Hercules, CA, USA). A filtered dilution of Oil Red O (Sigma-Aldrich, $0.3 \mathrm{~g}$ in $100 \mathrm{ml}$ of $60 \%$ isopropanol) was used on cells fixed with $4 \%$ formaldehyde and pictures were taken using an inverted microscope.

\section{Free glycerol determination in adipocyte cultures}

Concentration of excreted glycerol in the culture medium supernatant after the treatments was measured using a commercial assay kit (BioVision, Milpitas, CA, USA) according to the manufacturer's instructions and normalized to total protein content of the attached cells.

\section{F-actin quantification in adipocytes}

C3H10T1/2 were seeded on coverslips and differentiated into adipocytes. After treatments, cells were fixed with $4 \%$ formaldehyde, permeabilized with $0.05 \%$ Triton-X-100, and blocked with $2.5 \%$ bovine serum albumin. After washes, covers were incubated with $0.1 \mu \mathrm{g} /$ $\mathrm{mL}$ Alexa 568-phalloidin (Molecular Probes, Thermo Fisher Scientific Waltham, MA, USA) washed and nuclei were stained (ProLong Gold, Thermo Fisher Scientific. Fluorescence was analyzed under a confocal microscope TCS-SP5 (Leica Microsystems, Wetzlar, Germany). Phalloidin intensity was quantified and normalized to nuclei fluorescence using ImageJ software (NIH, USA).

\section{Animal models}

Animal experiments have been approved by the Institutional Animal Care and Use Committees from 
Universidad de Alcalá and Comunidad de Madrid (PROEX 230/16), in agreement with the guidelines established by the European Community Council Directives (2010/63/EU). Adult conditional ILK-deficient mice (cKDILK) were generated and subjected to diets as previously described [37]. Briefly, C57Bl/6 mice homozygous for floxed ILK flanked by loxP were crossed with BALB/c strain mice carrying a CMVdriven hydroxytamoxifen -inducible (TX, SigmaAldrich, St. Louis, MO, USA) CreER(T) recombinase gene globally expressed in all the tissues. 8-week-old male and females were injected intraperitoneal with $1.5 \mathrm{mg}$ of TX or vehicle, once per day for 5 consecutive days. 3 weeks after the injections, tail DNA was genotyped by PCR with primers corresponding to excised ILK gene (CCAGGTGGCAGAGGTAAGTA) or to nonexcised ILK (CAAGGAATAAGGTGAGCTTCAGAA ). The TX-treated mice displaying successful depletion of ILK were termed cKDILK, and the VH-treated without ILK depletion were termed wildtype (WT). Age-matched WT and cKDILK males and females were divided randomly and fed for 2 weeks either with a High Fat diet (HFD, $60 \mathrm{~kJ} \%$ fat, 8.46\% sucrose; D12492 Ssniff Spezialdiäten, Soest, Germany) or the corresponding low fat standard diet (STD, $13 \mathrm{~kJ} \%$ fat, $67 \mathrm{~kJ} \%$ carbohydrates, 10\% sucrose; Envigo Teklad Global Diet 2014, East Millstone, NJ, USA). Diets were given ad libitum during the time of the experiment. Each mouse had free access to water and was kept on a 12:12 h light:dark cycle at constant temperature of $21-23{ }^{\circ} \mathrm{C}$. The food intakes were calculated by subtracting the mass of food left from the initial food supplied. Along the period of diet challenges, TF (50 microg/KG, i.p.) or vehicle (saline) was administered daily [50]. After the experimentation conclude, animals were maintained under fasting conditions for $16 \mathrm{~h}$, weighted and euthanized with pentobarbital overdose. Body weight gains were calculated subtracting weights at the beginning from weights at the end of each experimental timeline. WAT depots and vastus lateralis were dissected and freshly processed or after preservation in RNAlater (Thermo Fisher Scientific, Waltham, MA, USA). TF pharmacokinetics determinations were performed in Rattus Norvegicus $(\mathrm{n}=6)$ after a single i.p. bolus of TF 50 microg/ $\mathrm{Kg}$. TF serological concentrations were determined in blood samples collected along $2 \mathrm{~h}$ after bolus and processed by liquid chromatography-mass spectrometry (LC-MS/MS).

\section{Reverse transcription-quantitative polymerase chain reaction (RT-qPCR)}

All products and equipment used were from Thermo Fisher Scientific. After the corresponding experiment, total RNA was extracted from cells or tissues collected from fasting mice. Equal amounts of RNA were transcribed to cDNA with HighCapacity cDNA RT Kit and $10 \mathrm{ng}$ of cDNAs were amplified using kits for qPCR. TaqMan gene expression assays were used to quantify MCP1 (Mm00441242_m1), IL6 (Mm00446190_m1), PPAR $\gamma, \quad$ (Mm00440940_m1), Leptin (Mm00434759_ $\mathrm{m} 1)$, FASN (Mm00662319_m1), FABP4 (00445878_ $\mathrm{m} 1), \quad$ HSL (Mm00495359_m1), ATGL (PNPLA2, Mm00503040_m1), AQP7 (Mm00431839-m1), FAT (Mm00432403_m1), UCP1 (Mm01244861_m1), PCNA (Mm05873628_g1) and ßactin (Mm01205647_g1). Amplification values were normalized to endogenous $\beta$-actin and relative quantification was determined with $2^{\wedge}-\Delta \Delta C T$ method. To quantify the non-excised ILK sequence between exons within the floxed area number 6 and 7 in WT and cKDILK specially designed primers (GGGCTCTTGTGAGCTTCTGT and GAGTGGTCC CCTTCCAGAAT) [29]. were determined with SYBR Green Master Mix and normalized to $\beta$-actin (GACGGC CAGGTCATCACTAT and CTTCTGCATCCTGTC AGCAA).

\section{Protein extraction and immunoblot analysis}

Cells or tissues were homogenized in lysis buffer $(10 \mathrm{mM}$ Tris-HCl, pH 7.6; 1\% Triton X-100; 1 mM EDTA; 0.1\% sodium deoxycholate) supplemented with protease and phosphatase inhibitors (Complete and PhosSTOP, Roche, Basel, Switzerland). Protein concentrations were determined by DC-Protein Assay (Bio-Rad, Hercules, CA, USA). Equal amounts were separated on SDSpolyacrylamide gels and transferred to $0.2 \mu \mathrm{m}$ - PVDF membranes (Bio-Rad, Hercules, CA, USA). Membranes were blocked, incubated with primary antibodies and secondary antibodies (Merck-Millipore, Billerica, MA, USA or Dako, Glostrup, Denmark) afterwards. Primary antibodies used were against AKT, P-AKT (Ser473), GSK3 $\beta$, P-GSK3 $\beta$ (Ser9), FAK, P-FAK (Tyr397), HSL, P-HSL (Ser660) (Cell Signaling Technology, Danvers, MA, USA), INTB1, P-INTB1 (Thr788/9) (Abcam, Cambridge, United Kingdom), Tubulin, Actin or GAPDH (Sigma-Aldrich, St. Louis, MO, USA). Immunoblots were detected by chemiluminescence (Pierce ECL Western Blotting Substrate, Thermo Fisher Scientific Waltham, MA, USA) and imaged with ImageQuant LAS 500 System (General Electric Healthcare, Little Chalfont, United Kingdom). Densitometries were measured using ImageJ software (NIH).

\section{Statistical analysis}

GraphPad Prism 5 Software was used to perform Student's $t$ test for 2 groups and 1- or 2-way analysis of variance form more groups, followed by Bonferroni's post 
hoc tests. Differences in mean values were considered statistically significant at a probability level of less than $5 \%(\mathrm{p}<0.05)$. Power of the study was $80-85 \%$, with a confidence level of $95 \%$.

\section{Results}

TF reduces TG content in the LD and the expression of adipogenesis markers in cultured adipocytes

Adipoblast-like C3H10T1/2 cells, which are a model for adipogenesis commitment studies [51], were cultured to confluency and induced to differentiate with specific mediums replaced every 2 days, as detailed in the methods section, until the complete differentiation on the $9^{\text {th }}$ day. To avoid the biological effects from serum and other growth factors present in the culture mediums while performing the treatments, fully differentiated cells were serum-deprived $24 \mathrm{~h}$ before and during TF (Aggrastat, Tirofiban $\mathrm{HCl} 50$ microg/ml, Correvio, UK) or vehicle (CT, physiological saline solution) treatments. TF concentrations used were in accordance with other in vitro experimental designs [47-49]. Figure 1 shows the quantification of TGs stored in LDs using lipid-specific dyes and the expression profile switch of some transdifferentiation markers after TF or CT treatment. Figure 1A shows representative microscopical pictures of adipocytes stained with the colorimetric lipidic dye Oil Red O. 50 microM TF reduces LD content per area after $24 \mathrm{~h}$ and modifies the apparent LDs distribution, from large LDs (as observed in CT) to smaller LD after TF treatment. Large LDs are characteristics of well differentiated adipocytes and WAT, meanwhile multilocular smaller LD are usually observed during transdifferentiation to non-adipogenic phenotype (pre-adipocyte, beige or brown adipocytes) in vitro [6]. Figure $1 \mathrm{~B}$ shows LD content reduction of approximately $30 \%$ loss after 50 or 100 microM TF for $24 \mathrm{~h}$, quantified with the fluorescent dye AdipoRed. Figure $1 \mathrm{C}$ shows that 50 microM TF-dependent LD content reduction appears as early as $4 \mathrm{~h}$ and significantly starts at $24 \mathrm{~h}$. The LD

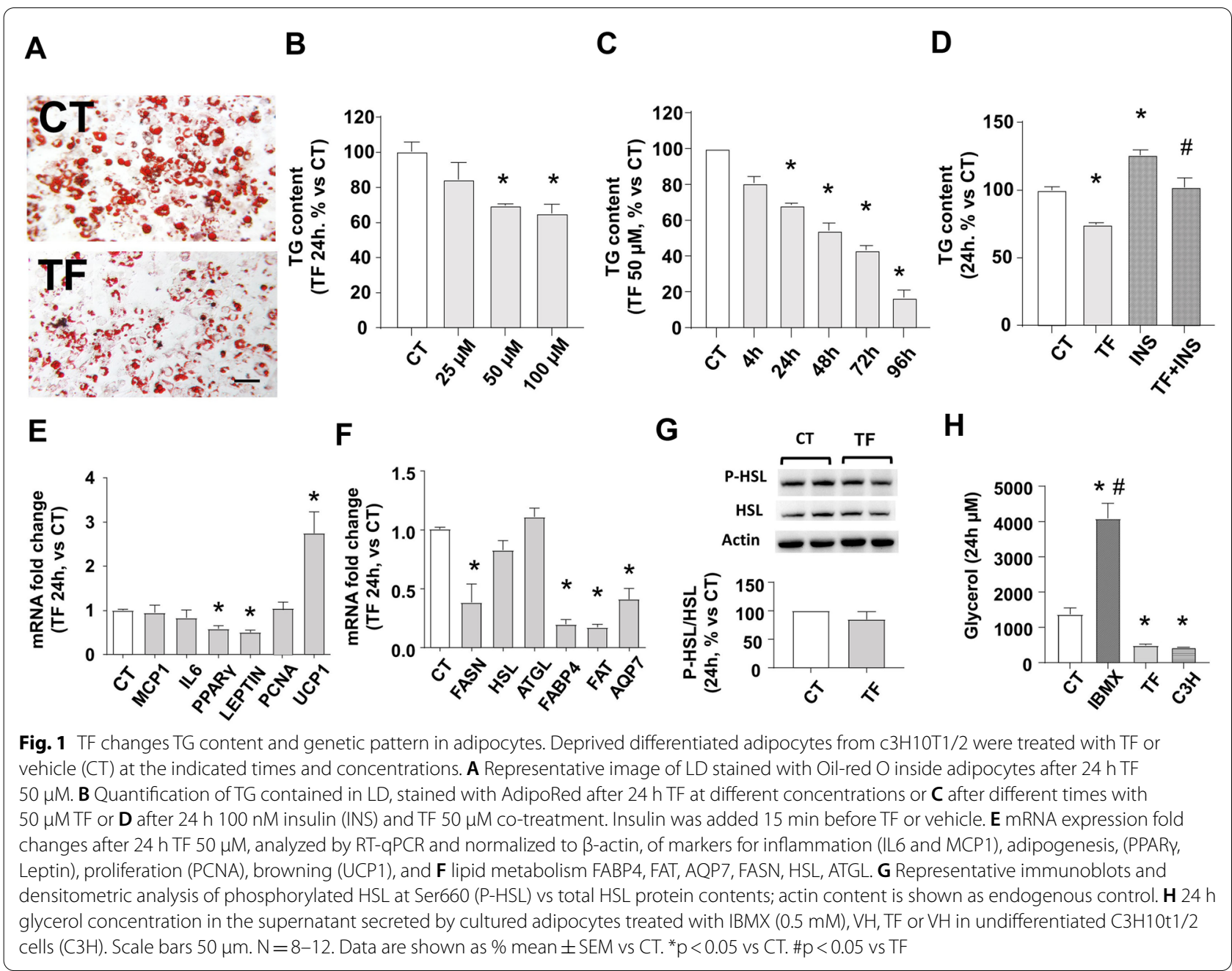


content reduction was accumulative reaching the lowest value (approximately 70\% loss) after $96 \mathrm{~h}$. From this point onward, we assessed for the rest of the in vitro experiments the use of 50 microM TF for $24 \mathrm{~h}$ as the minimal TF functional conditions. Figure 1D shows the TG quantification in $24 \mathrm{~h}$ serum-deprived adipocytes co-treated with TF and $100 \mathrm{nM}$ insulin for $24 \mathrm{~h}$, as a comparative study of serum deprived-cells versus a physiological-like condition. Insulin naturally promotes adipogenesis by increasing TG storage and reducing its expense by lipolysis [2]. Insulin stimulation in CT increases the LD content after $24 \mathrm{~h}$, as expected. However, TF prevented the insulin-induced $\mathrm{LD}$ content increase, reaching $\mathrm{CT}$ values. Increased pro-inflammatory adipocytokines, adipogenesis markers and intracellular TG content, are related with white adipocyte hypertrophy [2-5]. Figure $1 \mathrm{E}$ shows that TF treatment in cultured adipocytes does not modify the mRNA expression of pro-inflammatory adipocytokines MCP1 and IL6, while reducing adipogenesis markers (PPAR $\gamma$ and Leptin). TF so far inhibits hypertrophic signaling and does not affect WAT proliferation and hyperplasia, according to the unchanged expression of its marker PCNA [7]. TF increased UCP1, the thermogenic marker which is increased during white/ hypertrophied to beige/brown adipocyte transdifferentiation [8-12]. Other genes that are altered during adipocyte hypertrophy are fatty acid synthase (FASN), the lipolytic enzymes HSL and ATGL, and TG metabolite transporters FABP4, FAT and AQP7 [3, 13, 14]. Figure $1 \mathrm{~F}$ shows that TF downregulated FASN, FABP4, FAT and AQP7, but did not change HSL and ATGL. We further studied HSL activity and secreted glycerol to better study lipolysis during TF treatment. Figure $1 \mathrm{G}$ shows that 4 and $24 \mathrm{~h}$ of TF did not affect HSL phosphorylation at the crucial activation site Ser660. Secreted glycerol levels are shown in Fig. 1H. As a positive control, a single bolus of $0.5 \mathrm{mM}$ IBMX, which activates the PKA-dependent canonical lipolytic pathway, increases the secretion of glycerol in our settings. By contrast, neither VH nor TF-treated cells were able to increase glycerol. This is in accordance with the unaltered lipases expression and activity shown in the previous panels. Moreover, the steady state levels of glycerol secreted by TF-treated cells was even smaller than VH-treated ones, reaching the low levels of glycerol from the undifferentiated $\mathrm{C} 3 \mathrm{H} 10 \mathrm{t} 1 / 2$. The lowerthan-VH glycerol levels caused by TF emphasize its undifferentiating effect, which is probably due to the confluence of reduced TG content in the LD, reduced lipogenesis (FASN levels), unaltered lipolysis (lipases expression and activity), and reduced glycerol efflux (AQP7 levels).
TF binds to INT1B, allowing rapid intracellular phosphorylation and the rearrangement of the actin cytoskeleton

Figure 2A shows a rapid and significant INTB1 Thr788/9 phosphorylation [22] after TF treatment, starting at $15 \mathrm{~min}$ and maintained for at least $4 \mathrm{~h}$. Although TF was able to rapidly modify the phosphorylation of INTB1, its expression, determined as mRNA and protein content, was not modified $24 \mathrm{~h}$ later (Additional file 1: Figure S1A and B). To study the specificity of TF binding to the extracellular domain of INTB1, cells were pre-incubated with a very specific function-blocking INTB1 antibody (HMB1, Anti-mouse CD29 clone HMBeta1-1) prior to the treatment with TF or vehicle. This antibody antagonizes INTB1 efficiently during the outside-in mediation of other ligands [54]. Figure 2B shows that INTB1 blockade with HMB1 was able to avoid 60 min TF-mediated INTB1 phosphorylation. Figure $2 \mathrm{C}$ shows that HMB1 blocked the functional TF-mediated reduction of TG content $24 \mathrm{~h}$ later. Moreover, Fig. 2D shows that outsidein blocking tetrapeptide RGDS and -fiban drugs EPT and CIL were as efficient as HMB1 in blocking LD reduction mediated by TF-INTB1.

We followed the TF-INTB1 downstream regulation of actin cytoskeleton remodeling. Figure 3 shows the quantification of polymerized filamentous actin (F-actin) detected with phalloidin dye and normalized to nuclei present in the microphotography. TF disassembles (depolymerizes) $\mathrm{F}$-actin in as quick as $60 \mathrm{~min}$ (Fig. 3A) and is maintained for $24 \mathrm{~h}$ (Fig. 3B). The pretreatment with INTB1- blocking antibody HMB1 avoid the TF-dependent actin depolymerization. Conclusively, these results note TF as a modulator of INTB1-actin status, which can be antagonized by an INTB1 outside-in blockade.

\section{TF-INTB1-ILK axis: TF binding to INTB1 increases ILK expression and activity and modifies the expression of other markers to reduce TG content}

ILK is a main scaffold protein linking INTB1 and actin, and we studied its relevancy during TF-mediated transcriptional and functional. We blocked INTB1 and ILK pathways prior to TF treatment, by using the INTB1 blocking antibody HMB1 or ILK knockdown cells. To perform ILK knockdown in vitro, we transfected specific silencing RNAs (siRNA) against ILK (silLK). To assure that all the cells were under the same conditions, we used the same transfection conditions in all the experimentations shown in Fig. 4, with either control scrambled siRNA or silLK, as detailed in the methods section. Briefly, $48 \mathrm{~h}$ post-transfection, the cells were serumdeprived and treated with either HMB1 or vehicle $15 \mathrm{~min}$ before TF or vehicle treatments for $24 \mathrm{~h}$. Figure $4 \mathrm{~A}$ shows that TF increases the expression of ILK in cells 
A

\section{CT $15 \mathrm{~m} \quad 30 \mathrm{~m} \quad 60 \mathrm{~m} \quad 240 \mathrm{~m}$}
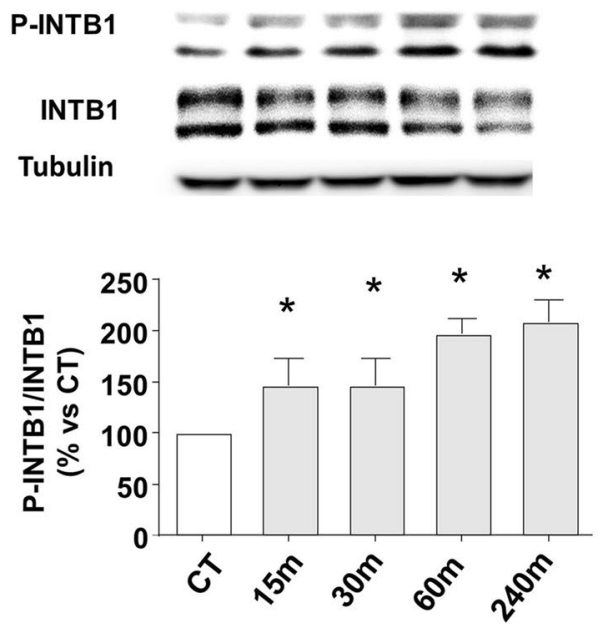

C

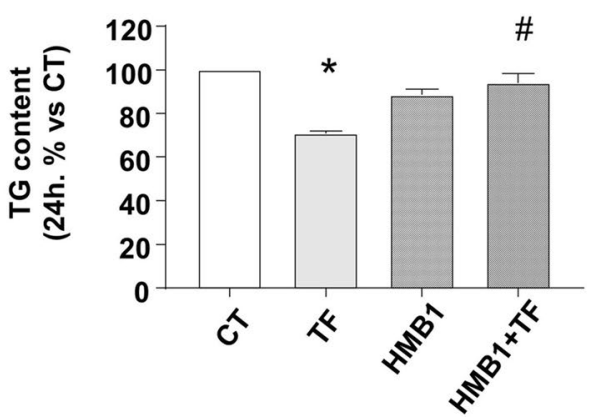

B
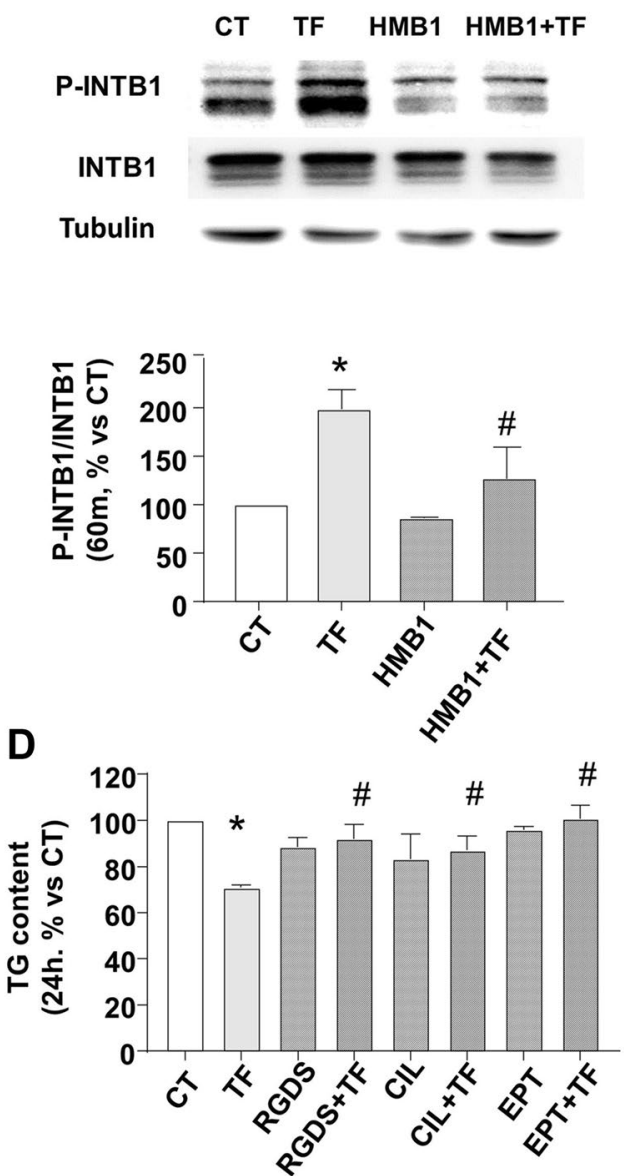

Fig. 2 TF interacts with INTB1 to reduce TG content in adipocytes. Deprived differentiated adipocytes from c3H10T1/2 were treated with TF $50 \mu M$ or vehicle (CT) or co-treated with a specific INTB1 blocking antibody (HMB1), RGDS, eptifibatide (EPT) or cilengitide (CIL) for the indicated times. $\mathbf{A}$, B Representative immunoblots and densitometric analysis of phosphorylated INTB1 at Thr788/9 (P-INTB1) vs total INTB1. Tubulin content is shown as endogenous control C, $\mathbf{D}$ quantification of TG contained in LD, stained with AdipoRed. $N=8-12$. Data are shown as mean $\pm S E M,{ }^{*} \mathrm{p}<0.05 \mathrm{vs} C T$, $\# \mathrm{p}<0.05$ vs TF

transfected with scrambled siRNAs. The ILK depletion in silLK was only partial in both vehicle and TF treated cells, reaching around $70 \%$ depletion. The partial knockdown of ILK by siRNAS transfection has been previously reported as common in mature adipocytes [52, 53]. Figure $4 \mathrm{~B}$ shows the consequences of ILK blockade on TG content. Cells transfected with scramble siRNAs and treated with TF have a similar TG downregulation as the non-transfected cells treated with TF, as shown in Fig. 1. Interestingly, the transfection with siILK significantly increased TG content compared to the control counterparts, which emphasizes the importance of the presence of ILK during the LD hypertrophy. On the other hand, TF was able to partially revert the TG increase caused by the partial depletion of ILK. We confirmed that ILK activity was increased in accordance with its expression after TF treatment, as measured by the phosphorylation state of well-known ILK downstream effectors AKT and GSK-3 $\beta$ [32]. Phosphorylation of AKT (Fig. 4C and Additional file 1: Figure S1C) and GSK-3 $\beta$ (Fig. 4D and Additional file 1: Figure S1D) were unaltered between $15 \mathrm{~min}$ to $4 \mathrm{~h}$, the time period of the fast INT phosphorylation and actin depolymerization is shown in Figs. 2 and 3. However, TF was able to increase the phosphorylation of AKT and GSK- $3 \beta$ after a longer time of exposure, beginning as early as $16 \mathrm{~h}$ and maintained at $24 \mathrm{~h}$, while the total content of both proteins was not affected. Figure 4E shows that INTB1 blockade with HMB1 partially avoid the TF-mediated increase of ILK expression. TF-dependent ILK activity shown as phosphorylation 
A
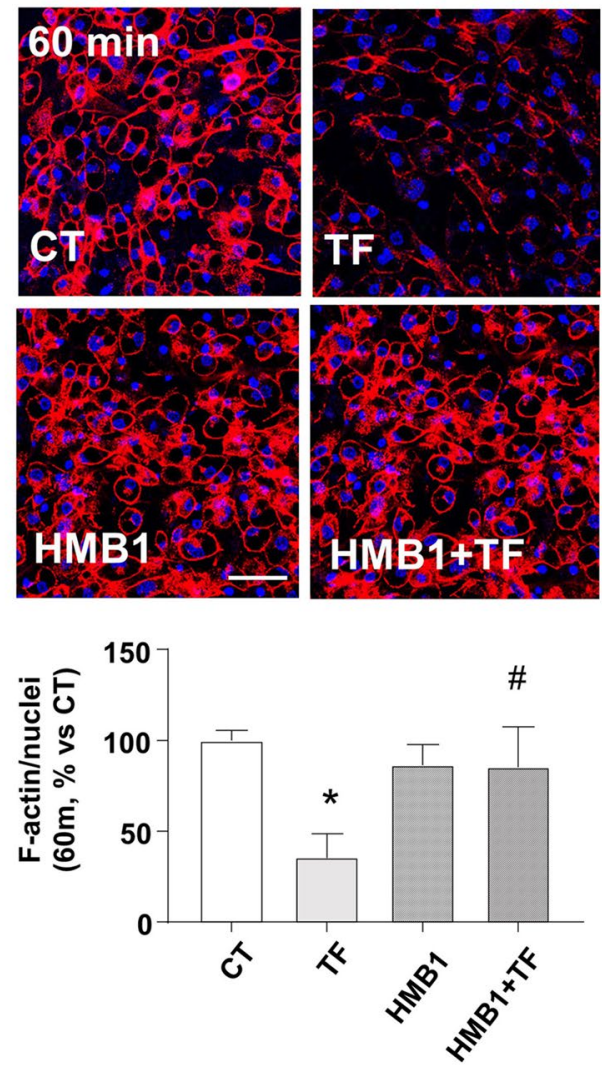

B
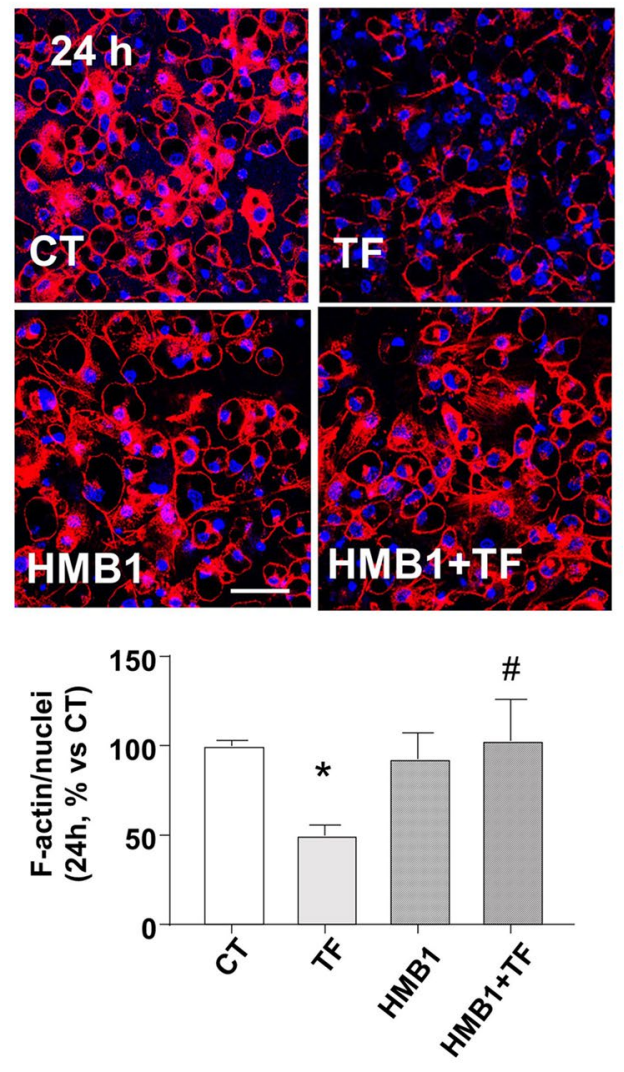

Fig. 3 TF reduces the levels of F-actin in adipocytes. Deprived differentiated adipocytes from c3H10T1/2 were treated with TF $50 \mu \mathrm{M}$ or vehicle (CT) or co-treated with specific INTB1 blocking antibody (HMB1) for the indicated times. Representative confocal microscopy images of F-actin dyed with phalloidin (red) and nuclei with DAPI (blue) and quantification of F-actin/nuclei per image after A 60 min and B 24 h of treatment. Scale bars $50 \mu \mathrm{m} . \mathrm{N}=8-12$. Data are shown as mean $\pm \mathrm{SEM},{ }^{*} \mathrm{p}<0.05$ vs $\mathrm{CT}$, \#p $<0.05$ vs TF

levels of AKT in Fig. 4F, was increased and partially blunted after INTB1 blockade, in accordance with the ILK expression levels. Furthermore, we studied the role of the INTB1-ILK axis driven by TF during the transcription of transdifferentiation genes, as already shown in Fig. 1. The blockade of INTB1 and ILK revert completely the TF-mediated expression changes on some representative markers, as PPARY (Fig. 4G) and UCP1 (Fig. 4H). The effect on transcription was complete, unlike the partial effects observed during LD content (Fig. 4B) and ILK expression (Fig. 4E). Conclusively, TF uses INTB1ILK axis to reduce TG content and to change the transcription of related genes. The axis blockade partially reverts the effect on TG content and ILK expression, but completely revert the transcription of other markers. The incomplete TF-mediated reversion of LD content in silLK-transfected cells can be interpreted as the participation of another ILK-independent mechanism, but it can be also considered the non-depleted ILK presence in silLK cells, where approximately $30 \%$ of ILK remains expressed and functionally modulated by TF to decrease partially the TG content. The partial transcriptional regulation of ILK when using HMB1 suggests the participation of a TF-INTB1-independent mechanism during ILK transcription, but not in the transcription of PPAR $\gamma$ and UCP1. Besides ILK, another kinase that is noted to be downstream INTB1 is FAK $[31,55]$. TF does not modify FAK protein content nor its activity, which is measured as Tyr297 phosphorylation (Additional file 1: Figure S1E).

\section{In vivo approach: TF systemic administration prevents} obesity appearance in a HFD-based mice model, increases ILK and modifies other markers expressions in visceral AT TF pharmacokinetics are similar in humans and rodents, with an early clearance of up to $2 \mathrm{~h}$, and it is clinically approved as anticoagulant for a maximum of $18 \mathrm{~h}$ of continuous intravenous perfusion of 25 microg/ $\mathrm{kg}$. [56]. To observe functional effects in rodents, systemic 


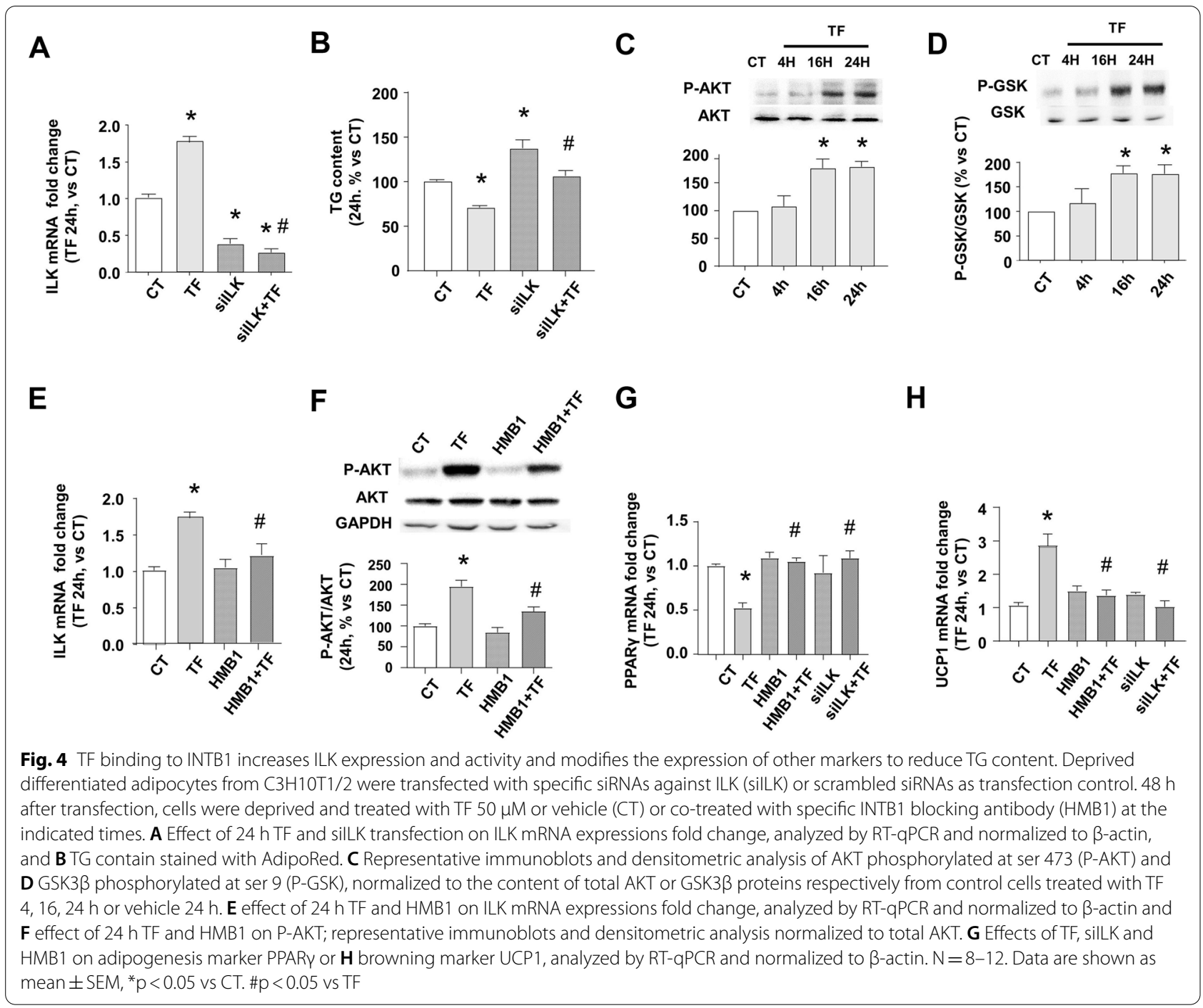

administration of TF 50 microg/Kg body weight can be performed intraperitoneally (i.p.) [42-50]. In this regard, we first studied the pharmacokinetics of 6 rodents (Rattus Norvegicus) after a single i.p. bolus of TF 50 microg/ $\mathrm{Kg}$. To determine TF serological concentrations, blood samples were collected at different times and processed by LC-MS/MS. The maximum peak of TF was reached $15 \mathrm{~min}$ after i.p. and rapidly dropped to undetectable values after $2 \mathrm{~h}$ (mean values of TF in $\mathrm{ng} / \mathrm{ml}$ of serum: time 0 control 0.39; 15 min 19.87, 30 min 15.73, 45 min 6.03, $60 \min$ 6, $90 \mathrm{~min} 5.36,120 \mathrm{~min}$ 0.51). To further elucidate the prolonged use of TF during the early establishment of obesity in vivo and the involvement of ILK, we designed a transgenic mice model $[36,37]$ with a global ILK downregulation during adulthood (cKDILK). These animals and their control counterparts (WT) without ILK depletion were divided to be challenged with either HFD or STD, the latter serving as control. HFD-challenged WT allowed us to follow TF consequences on the establishment of obesity, while HFD-challenged cKDILK are a transgenic tool to understand the relevance of ILK. We previously demonstrated that short-term HFD administration in mice for 2 weeks allows for the early establishment of obesity and insulin-resistance and for observation of ILK involvement [36]. Considering this and TF pharmacokinetics, we administered a single daily bolus i.p. of 50 microg/Kg TF per day in the mice throughout the 2 weeks of diet challenges. At the end of the experiments, mice were sacrificed, and WAT depots were dissected, weighed, and normalized to total body weight (BW). No difference in daily food or water intakes was observed between WT and cKDILK, nor between $\mathrm{VH}$ and TF-treated mice subjected to the same diet (Additional file 1: Table S1). The food grams intake was lower in VH HFD groups (WT or cKDILK) compared to VH STD counterparts, although the consumption of 


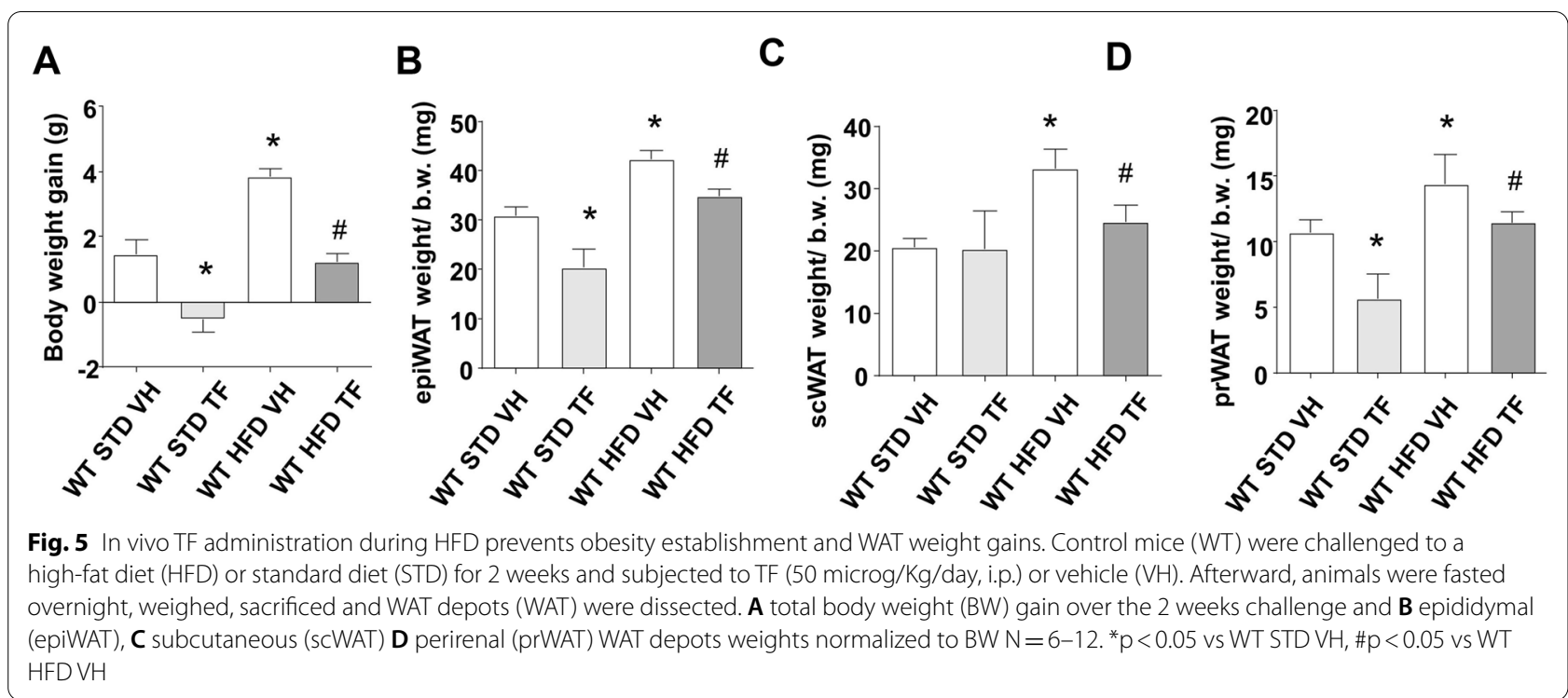

calories is increased due to HFD composition. Interestingly, food and water intakes of TF groups were not different to VH STD. Figure 5A shows BW gains between the beginning and the end of the diet/treatment challenge. HFD increased efficiently BW, despite the lower food intake, as we already published in our previous work [36]. Interestingly, TF treatment during this period reduced $\mathrm{BW}$ gain when compared to $\mathrm{VH}$, in both $S T D$ and HFD challenged WT. Figure $5 \mathrm{~B}-\mathrm{D}$, show, respectively, the weights of epididymal (epi), subcutaneous (sc) and perirenal (pr) WAT depots, normalized to BW, from the same animals. TF reduced epiWAT and prWAT ratios, but not scWAT in STD WT. All the WAT ratios were increased in $\mathrm{VH}$-treated HFD WT, but were reduced in TF-treated HFD WT, in accordance with the BW gain changes shown in Fig. 5A. Figure 6A shows that TF increased ILK expression in epiWAT from both STD and HFD WT. INTB1 expression was unmodified in vivo and in vitro, while the phosphorylation in Thr788/9 did not persist after TF prolonged treatment in vivo, compared to the quick phosphorylation observed in vitro (Additional file 1: Figure S1F). The TF-dependent ILK upregulation in epiWAT from mice under physiological conditions and fed with STD mostly occurs in the main cell population present in WAT, which consists of adipocytes and pre-adipoctyes, although other cell types may be present to a lesser extent. However, under obesogenic HFD conditions, epiWAT is hypertrophied and the infiltrated macrophages population is increased, although the adipocytes-like population is the majority $[1,5,6$, 29]. We already published that ILK is downregulated in epiWAT from HFD [36] and TF was able to revert this to control (STD) values. Despite in vitro adipocyte ILK expression being upregulated by TF as in vivo (epiWATs from either STD or HFD), we wondered whether TF may change ILK expression in other cell types besides the adipocytes-like populations present mostly in WAT, such as macrophages, or even in other tissues, such as the skeletal muscle. Using the same conditions as with the cultured adipocytes, an in vitro approach of TF treatment was performed in macrophage cell line THP-1 and myocyte-like $\mathrm{C} 2 \mathrm{C} 12$. TF was not able to change ILK expression in THP1 (Additional file 1: Figure S2A), C2C12 (Additional file 1: Figure S2B) and the skeletal muscle (vastus lateralis) from TF-treated HFD (Additional file 1: Figure S2C). Because therapeutic use of TF may be conceptually important in HFD hypertrophied WAT, we simplified the next panels by only exposing the results from HFD-challenged mice. Figure 6B shows that TF increases AKT and GSK3 $\beta$ phosphorylation in epiWAT from HFD. Figure $6 \mathrm{C}$ shows that TF reduces the mRNA expression of upregulated markers MCP1, IL6, PPAR $\gamma$ and leptin present in the hypertrophied epiWAT [1, 2, 23]. Proliferation marker PCNA was not modified. The transdifferentiation of beige adipocytes within WAT to brown-like phenotype is exhibited by higher levels of UCP-1, directly related to increased lipid metabolism and thermogenesis. [10]. Beige adipocytes exist mainly in scWAT rather than other visceral depots (e.g. epiWAT) in rodents [11, 12]. Figure $6 \mathrm{C}$ shows that TF did not increase UCP-1 in the epiWAT of HFD-mice, probably due to its limited browning capacity. We further studied in Fig. 6D the phenotypically more flexible scWAT inguinal depot, where ILK and UCP-1 were overexpressed after TF, which correlate with the trans-differentiation pattern observed in cultured adipocytes and suggesting a thermogenesis-mechanism 
A

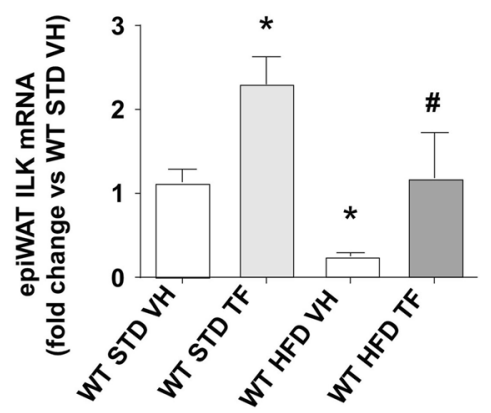

C

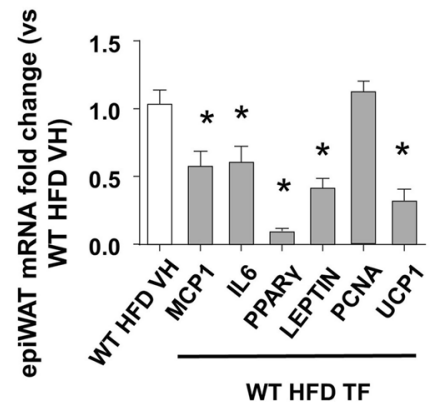

D

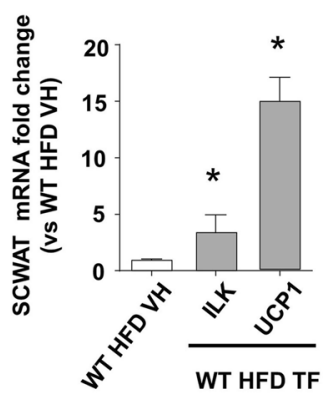

B

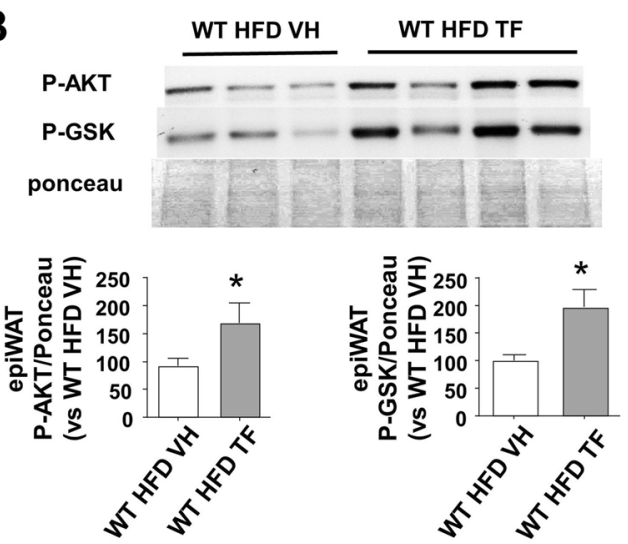

E

Fig. 6 In vivo TF administration during HFD upregulates ILK in WAT and modifies the genetic pattern of several markers in WAT depots. Control mice (WT) were challenged to a high-fat diet (HFD) or standard diet (STD) for 2 weeks and subjected to TF (50 microg/Kg/day, i.p.) or vehicle (VH). Afterward, animals were fasted overnight, weighed, sacrificed and WAT depots (WAT) were dissected. A Fold changes of ILK mRNA expression analyzed by RT-qPCR and normalized to $\beta$-actin in epiWAT from STD and HFD WT. B Representative immunoblots and densitometric analysis of AKT phosphorylated at Ser473 (P-AKT) and GSK3 $\beta$ phosphorylated at Ser9 (P-GSK), normalized to total protein content (transference membrane dyed with ponceau) in epiWAT of HFD WT. C HFD WT epiWAT mRNA expression fold changes, analyzed by RT-qPCR and normalized to $\beta$-actin, of markers for inflammation (IL6 and MCP1), adipogenesis, (PPARY, Leptin), proliferation (PCNA), browning (UCP-1). D HFD WT scWAT mRNA expression fold changes of ILK and UCP1. E HFD WT epiWAT mRNA expression fold changes of lipid metabolism markers FABP4, FAT, AQP7, FASN, HSL, ATGL. $\mathrm{N}=6-12 .{ }^{*} \mathrm{p}<0.05$ vs WT STD VH, \#p $<0.05$ vs WT HFD VH

in scWAT to lose energy and weight gain. On the other hand, epiWAT weight gain in HFD-mice is mostly caused by hypertrophy, thus increased lipogenesis and/ or reduced lipolysis of white adipocytes [1-4]. Figure $6 \mathrm{E}$ shows that lipogenesis marker FASN was reduced in TFtreated mice, while lipase HSL was increased. ATGL, FABP4 and AQP7 remained unmodified, while FAT was increased. Altogether, these data indicate active lipolysis in epiWAT to reduce its weight gain.

\section{The lack of ILK in WAT avoids the anti-obesogenic effect of TF}

We further studied the relationship between BW gains and ILK presence in epiWAT by using CKD-ILK challenged to diets and i.p. treatments as their WT counterparts. Figure 7A, B show BW gains of STD andr HFD-challenged animals, respectively. Under both diets,
TF was not able to reduce BW gains in cKDILK as happens in WT. Moreover, Fig. 7B also shows that HFD cKDILK BW gain is even higher than that of HFD WT, something that has already been observed in our previous work [36]; however TF was not able to minimally decrease this BW gain. Figure $7 C$, D show the efficient ILK depletion in the epiWAT of cKDILK when compared with that of WT in both STD and HFD. The ILK expressions are in accordance with $\mathrm{BW}$ gains. In both cases, TF was not able to revert the ILK downregulation in cKDILK, while in TF treated WT, the increase of ILK expression was clearly shown (Fig. 5). Figure 8 summarizes a putative INTB1-ILK mediated mechanism of TF in WAT adipocytes. ILK presence is relevant during obesity establishment. TF pharmacologically transdifferentiates the adipocytes within hypertrophied WAT and therefore avoid obesity. TF uses the axis formed by 
A

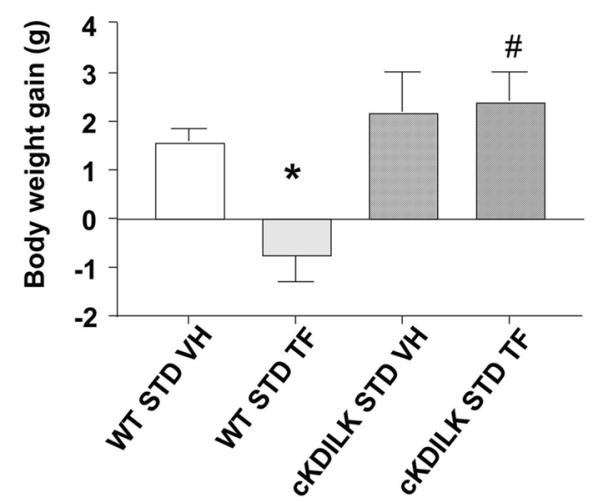

C

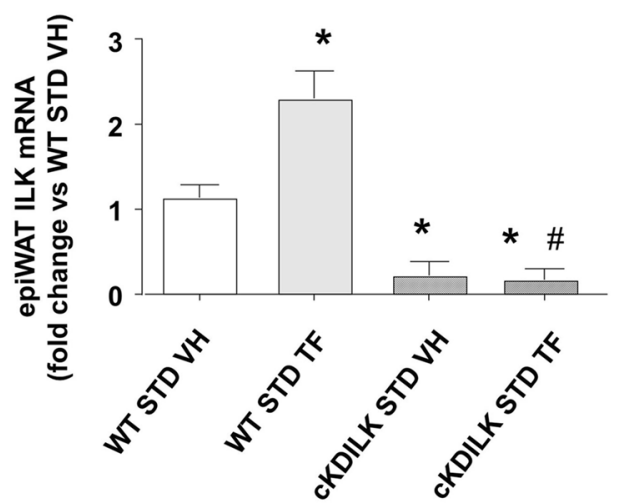

B

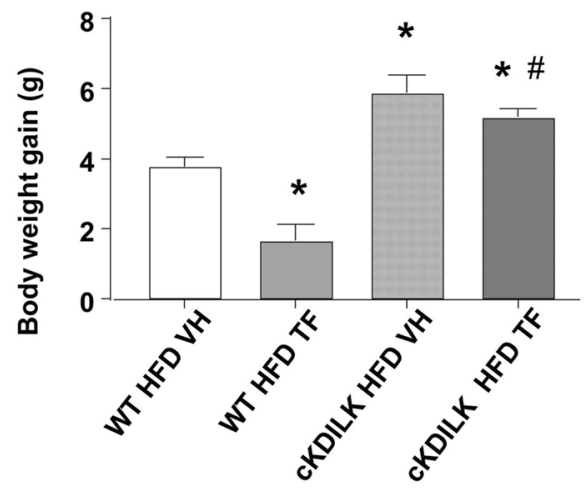

D

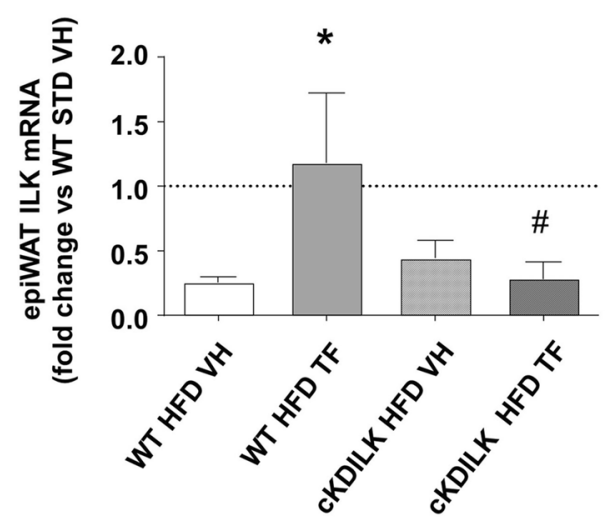

Fig. 7 In vivo TF administration on ILK depleted cKDILK does not prevent obesity. Conditional Knockdown ILK (cKDILK) or control wildtype counterparts (WT) were challenged to a high-fat diet (HFD) or standard diet (STD) for 2 weeks and subjected to TF (50 microg/Kg/day, i.p.) or vehicle (VH). Afterward, animals were fasted overnight, weighed, sacrificed and epididymal white AT depots (epiWAT) were dissected, weighed, and processed accordingly. A, B total body weight (BW) gains over the 2 weeks challenge in STD and HFD, respectively. C, D fold changes of ILK mRNA expression analyzed by RT-qPCR and normalized to $\beta$-actin in STD and HFD, respectively. N =6-12. ${ }^{*} p<0.05$ vs WT VH, \#p $<0.05$ vs WT TF

INTB1-ILK-actin in the adipocytes to change lipidic metabolism, intracellular TG content and adipocytic and ILK genes transcription.

\section{Discussion}

Obesity is one of the major global health concerns, even though only a few medications have been approved for the reduction of energy intake, its absorption or the promotion of its expenditure. Due to the long-term side effects, particularly cardiovascular issues, new antiobesity drug development must be focused on both weight loss efficacy and cardiovascular safety [50]. In this study, we demonstrated the unexpected use of clinically approved TF as a potential anti-obesogenic. TF is particularly relevant as cardiovascular protector because it is an anticoagulant with vasodilator and endothelial protective properties [42-50]. Moreover, other RGD-based "-fiban" drugs were developed as vascular protective "superaspirines" [39, 40]. Our approach has never been considered for other accepted anti-obesogenic drugs: TF reduces adipogenesis in both cultured adipocytes and hypertrophied WAT from diet-induced obese mice. To target INTB1 signaling appears to be a logical method to alter adipogenesis and prevent obesity, since hypertrophic adipocytes have upregulated levels of INTB1 and there is an intimate relation between INTB1 and adipogenesis [5, 18-21]. However, almost no attention had been given to INT1B modulation in AT malfunction until the development of this study, although the potentiality of these agents as therapeutics in other applications is patent [9]. The importance of INTs during the development of adipose tissue has been emphasized with the use of transgenic knockout mice models. However it is not a good approach for the study of obesity. Indeed, the complete knockout of INTB1 is lethal [18] and the adipose-selective conditional deletion of INTB1 produces 

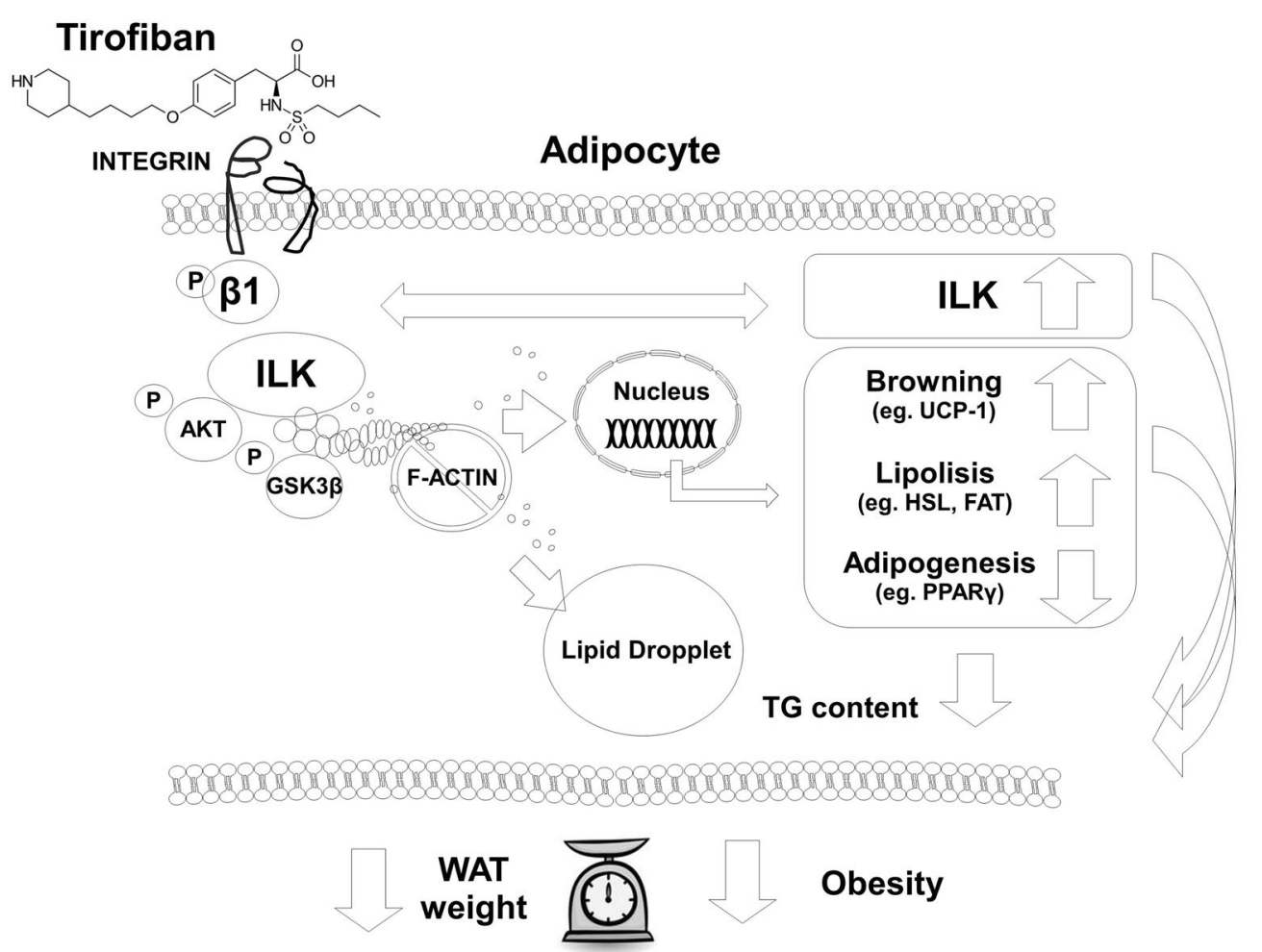

Fig. 8 Putative mechanisms of TF-INTB1-ILK action. TF binds to adipocyte INTB1, depolymerizes F-actin and reduces the TG content on the lipid droplet. Adipogenesis-related genes are transcriptionally downregulated while lipolysis, thermogenesis and ILK genes are upregulated. ILK presence is part of the gear for TF function. Therefore, WAT adipocytes transdifferentiate to catabolic/beige phenotype to reduce WAT hypertrophy and obesity

an atypical lipodystrophy [19]. On the other hand, the knockout of INT subunit alpha4, an INTB1 partner in adipocytes, can ameliorate HFD-based obesity [16]. In accordance with these premises, here we demonstrated that the pharmacological interaction of TF specifically with INTB1 ameliorated obesity, and the use of known outside-in blockers such as HMB1, RGDS, CIL and EPT $[39,40,54]$ blocked the effect of TF while they were not able to reduce TG content by themselves. TF interacted with INTB1 and produced rapid phosphorylation of cytoplasmic Thr788/9, which is part of inside-out rather than outside-in signaling [22], but due to the fine-tuned balancing act between inside-out structural modulation and outside-in functional modulation, further research may be devoted to understanding the rapid phosphorylation of INTB1 as well as which adaptor proteins are involved during this early inside-out signaling. We found some of the mechanisms followed by the TF-INTB1 interaction, however, we did not completely list the sequence of events, because adipocyte transdifferentiation occurs due to an intimate and not well-understood relationship between cytoskeletal rearrangement, TG storage/expenditure, LD configuration (size, locularity, morphology), metabolite transport dynamics and transcriptional regulation [15, 23-27]. In vitro, TF reduced LD size, increased multilocular LD configuration and the expression of pre-adipoctye and/or beige adipocyte markers, in accordance with anti-hypertrophic transdifferentiation [19]. In WAT from animals treated with $\mathrm{TF}$, transdifferentiating mechanisms of lipolysis and/ or browning were also patent. It is not easy to track and interpret the exact process of communication between TF-INTB1 and the downstream cytoskeletal rearrangement, because the modulation of INTB1 during WAT differentiation or dysfunction is highly complex, not linear and involves a vast array of enzymes, adaptors, and cytoskeletal components. [5, 8, 9, 18-21]. Several inconsistencies are reported concerning the remodeling and dynamics of each cytoskeleton component (e.g., actin, microtubules) during adipocyte maturation, due to variable experimental conditions (e.g. cultured cell model, AT depots used from animal models, intracellular location of the network, etc.) [24, 27]. For example, adipocyte hypertrophy correlates with increased F-actin in some of these reports $[20,25]$ and its disruption reduces adipose differentiation [57], while other reports suggest opposite 
observations, that is, adipogenesis is enhanced when cytoskeleton components are disrupted [26, 58-60].

The use of INTB1-blocking agents demonstrated that TF-mediated rapid and persistent actin depolymerization is part of the specific INTB1 outside-in signaling started by TF in correlation with the reduction of LD content. TF reduced F-actin levels in vitro, but that does not necessarily mean that the cytoskeleton is completely disrupted, unestablished, or that its dynamic is blunted. Actin depolymerization mediated by TF which extends from the cytoplasm to the nucleus, may be the mechanical base for the transcriptional modulation during adipocyte differentiation, as demonstrated in several cell types $[61,62]$. Our work is distant to completely clarify TF-driven transmission between actin and the nuclear transcription. Nevertheless, actin depolymerization by TF is expected to be followed by the adipocytes within WAT, as demonstrated by comparative studies of cultured adipocytes vs whole tissue WAT [63]. We used two experimental approaches to address our hypothesis, a single dose of TF in cultured adipocytes and HFD-based obesogenic model in vivo with systemic TF administration for several days. The evident differences may result in differences of expressions and mechanisms followed inside the adipocytes. TF did not increase pro-inflammatory cytokines expression in vitro, an expected safety concern already addressed since TF is an approved drug. Interestingly, TF reduced these cytokines in WAT from in obesogenic (HFD) mice, which gives an advantageous effect of TF by reducing WAT inflammation that causes malfunction $[1,5]$. Adipocyte hypertrophy is upregulated during obesogenesis and can be tracked by the expression of PPARy and leptin, which were both blunted by TF. TG content (LD size) reconfiguration in vitro may be based on reduced lipogenesis rather than increased lipolysis: a) TF reduces LD content despite the stimulation of anti-lipolytic insulin [2], b) lipogenesis marker FASN was reduced and b) neither the expression of lipases, nor HSL activity [4] were increased in cultured adipocytes treated with TF, while the pivotal glycerol efflux channel AQP7 [13, 14] and therefore the steady state glycerol secretion outside the cells were downregulated. Moreover, the TF-mediated lower-than-VH basal steady-state glycerol secretion reached undifferentiated C3H10T1/2 levels, which also may indicate the grade of undifferentiation reached by TF-treated adipocytes. On the other hand, long term in vivo use of TF increased the expression of lipolysis markers HSL and FAT in epiWAT. Therefore, it is plausible that TF uses lipolysis in vivo, as part of the mechanisms to reduce hypertrophic visceral WAT weights in $\operatorname{HFD}[1,2,4]$. TF increased thermogenic UCP1 [8-12] while decreased
AQP7 in vitro, pointing a white-to-brown transdifferentiation. AQP7 downregulation is considered another beige adipocyte differentiation marker, because the heat production from oxidative metabolism related to the beige adipocytes contrasts with the anabolic/catabolic lipid metabolism requiring glycerol gateways occurring in white adipocytes $[13,14]$. In TF-treated mice, UCP-1 expression was increased in scWAT, which is profuse in beige adipocytes [10-12], although UCP1 and AQP7 were not modified in epiWAT. Increased thermogenesis in scWAT was therefore the responsible pathway followed to produce the weight loss. Conclusively, food intakes were the same between HFD groups, but TF increases the expenditure of energy overload by rather increasing WAT depotdependent lipolysis and/or thermogenesis. We found the relevant role of ILK during hypertrophy in adipocytes because ILK loss produces an aggravation of the hypertrophy inside the adipocytes and in the WAT weights. By using TF, we demonstrated that increase of ILK yield in WAT is protective against obesity, and the ILK upregulation was specific for adipocytes-like cells within the WAT and it was not observed in other TFtreated cell types and/or tissues. The blockade of INTB1 with HMB1 and the use of cells without ILK (silLK) demonstrate the importance of TF-INTB1-ILK transmission of the transcriptional and functional effects. INTB1 blockade was able to completely revert the TF-mediated TG content change, as well as the actin depolymerization. HMB1 completely revert TFmediated PPAR $\gamma$ and UCP1 transcription while ILK upregulation was only partially reverted, probably due to the participation of different mechanisms (e.g., Transcriptional factors). ILK blockade (siILK) also reverted completely the TF-mediated transcription of PPAR $\gamma$ and UCP1, while the reversion of LD downregulation was partial, which can be interpreted as the participation of an ILK-independent mechanism, or it can be also considered the presence in silLK cells of approximately $30 \%$ non-depleted ILK, which remains functional and therefore can be modulated by $\mathrm{TF}$ to decrease only partially the TG content. We studied the ILK effects in vivo, by using a transgenic model of global ILK depletion in the adulthood (cKDILK). Our previous works showed that cKDILK under HFD, where ILK is successfully under-expressed in WAT, are predisposed to gain weight and insulin resistance [36, 37], and therefore can be considered a better tool than other knockouts for upstream or downstream elements. Together with the present work, our results support the hypothesis that ILK expression is decreased during the development of obesity and WAT transgenic downregulation of ILK (in cKDILK) exacerbates the 
obesity-related symptoms. The opposite hypothesis has been stated in Bugler-Lamb's work [38], where ILK expression was increased by a long-term HFD and the use of adipocyte-specific ILK-deficient mice displayed reduced fat mass and improvement of glucose tolerance, as the in vitro knockdown of ILK in 3T3-L1 cells decreased lipid accumulation in their work. The differences of both methodological and conceptual approaches between Bugler-Lamb's models and ours are several and may explain the apparently contradictory results between studies. Nevertheless, we did carry out a pharmacological strategy to increase ILK expression in vitro and in vivo as a protective approach against obesity that was not used in Bugler-Lamb's study. The ILK upregulation is accompanied by the increased activity of ILK, measurable by the phosphorylation of downstream effectors such as AKT at Ser473 as well as GSK3 $\beta$ at Ser9. AKT/GSK3 $\beta$ phosphorylation, and by extension ILK activity, were not as rapid as INTB1 phosphorylation in vitro. Therefore, these kinases were probably not responsible for the rapid inside-out TF-mediated INTB1 phosphorylation. Other kinases have been noted as adaptor enzymes during adipocytic differentiation, such as mitogen-activated protein kinase/extracellular signal-regulated kinase, PI3K, Rho-associated protein kinase, AMP-activated protein kinase, and the Src kinases family [28-30]. Particularly, FAK is usually referred to as being placed upstream in the hierarchy of recruitment and activation of those kinases, including AKT during INTB1 modulation [31]. Our results showed that FAK activity, analyzed by phosphorylation at Tyr397, was not altered throughout the TF treatment time-lapse. Thus, it is probable that FAK or related downstream kinases are not relevant during the TF-mediated effects. Although ILK activity may not be responsible for upstream INTB1 phosphorylation, our results indicate that an axis made up of ILK-AKT/GSK3 $\beta$ may be responsible for the downstream TF-mediated reduction of adipogenesis and hypertrophy. Some publications agree with this hypothesis: AKT and GSK3 $\beta$ are related to adipocyte differentiation [28-30] and, as we already demonstrated, cKDILK animals have reduced AKT phosphorylation in epiWAT, concomitantly with the downregulated ILK expression and the increased fat mass and insulin resistance $[36,37]$ as well as TF depends on AKT to favor endothelial cell growth [42, 43]. AKT/GSK3 $\beta$ showed phosphorylation after a longer period of TF treatment (16/24 h in vitro, 2 weeks of daily administration in vivo), in accordance with the observed increased expression of ILK, however, it is also probable that the repeated and long-time exposure of TF in vivo produces an indirect effect on AKT/
GSK3 $\beta$. Nevertheless, to address the role of each abovementioned kinases (besides ILK) during TF-mediated adipocyte transdifferentiation, further experimental models may be necessary (e.g. Specific silencing of each kinase).

\section{Conclusions}

We consider ILK expression levels in WAT to be a biomarker of metabolic malfunction during the development of obesity. Pharmacological treatments able to increase ILK expression are a new strategy to restore the malfunctioning WAT. TF is a well-established anticoagulant with known cardiovascular protective benefits. TF increases ILK expression and activity and reduces adipogenesis and facilitates adipocyte transdifferentiation. The consequence is that TF administration in vivo reduces WAT hypertrophy and weight gain. These preclinical results provide the proof of concept for more translational studies to design a cardiovascular safe treatment based on TF-mediated interaction of INTB1-ILK.

\section{Abbreviations}

AKT: Protein kinase B; AQP7: Aquaglyceroporin 7; ATGL: Adipose Triglyceride Lipase; BW: Body weight; CIL: Cilengitide; CKDILK: ILK knock-down mice; CT: Control, vehicle-treated cells; ECM: Extracellular matrix; epiWAT: Epididymal WAT depot; EPT: Eptifibatide; F-actin: Polymerized filamentous actin; FABP4: Fatty Acid Binding Protein 4; FAK: Focal adhesion kinase; FASN: Fatty Acid Synthase; FAT: Fatty Acid Translocase; FBS: Fetal bovine serum; GSK3 $\beta$ : Glycogen synthase kinase $3 \beta ;$ HFD: High fat diet; HMB1: Anti-mouse CD29 clone HMBeta1-1, INTB1 blocking antibody; HSL: Hormone Sensitive Lipase; IBMX: 3-Isobutyl-1-methylxanthine; IL6: Interleukin-6; ILK: Integrin-linked kinase; INS: Insulin; INT: Integrin; INTB1: Integrin- $\beta 1$; LC-MS/MS: Liquid chromatographymass spectrometry; LD: Lipid droplets; MCP1: Monocyte chemoattractant protein-1; PCNA: Proliferating cell nuclear antigen; prWAT: Peri-renal WAT depot; RGD: Arg-Gly-Asp peptide; RGDS: Arg-Gly-Asp-Ser peptide; scWAT: Subcutaneous WAT depot; silLK: Silencing RNA against ILK; siRNA: Silencing RNA; STD:

Standard diet; TF: Tirofiban; TG: Triglycerides; TX:Tamoxifen; UCP1: Uncoupling Protein-1; WAT: White adipose tissue; WT: Wildtype mice.

\section{Supplementary Information}

The online version contains supplementary material available at https://doi. org/10.1186/s13578-022-00746-1.

Additional file 1: Table S1. Food and water intakes during experimental conditions in vivo. Conditional Knockdown ILK (CKDILK) or control wildtype counterparts (WT) were challenged to high fat diet (HFD) or standard diet (STD) for 2 weeks and subjected to TF (50 microg/ $\mathrm{Kg} /$ day, i.p.) or vehicle $(\mathrm{VH})$. Food and water intakes per animal were measured every day along the experiment. Values are represented as mean $+/-$ SEM. $N=6-12 .{ }^{*} p<0.05$ vs STD. Figure S1. TF does not modify INTB1 and FAK expressions after $24 \mathrm{~h}$, phosphorylation of AKT, GSK and FAK from 15 to $240 \mathrm{~min}$, FAK at $24 \mathrm{~h}$ or INTB1 after 2 weeks administration in vivo. A-E Deprived differentiated adipocytes from c3H10T1/2 were treated with TF $50 \mu \mathrm{M}$ or vehicle (CT) for the indicated times. A) $24 \mathrm{~h}$ fold changes of INTB1 mRNA expression were analyzed by RT-qPCR and normalized to $\beta$-actin B), Representative immunoblots and densitometric analysis of total INTB1 levels after $24 \mathrm{~h}$, normalized to tubulin levels. C, D Representative immunoblots and densitometric analysis of AKT phosphorylated at Ser473 (P-AKT) and GSK3 $\beta$ phosphorylated at Wer9 (P-GSK) respectively, between 15 and 240 min and normalized to total AKT or 
GSK3 $\beta$ protein contents. E Representative immunoblots and densitometric analysis of FAK phosphorylated at Tyr297 (P-FAK) between $15 \mathrm{~min}$ and $24 \mathrm{~h}$ and normalized to total FAK protein content. F Control mice (WT) were challenged to high fat diet (HFD) for 2 weeks and subjected to TF (50 microg $/ \mathrm{Kg} /$ day, i.p.) or vehicle (VH). Afterward, animals were fasted overnight, weighed, sacrificed and epididymal white AT depots (epiWAT) were dissected and processed. Representative immunoblots and densitometric analysis of phosphorylated INTB1 at Thr788/9 (P-INTB1) vs total INTB1. Data are shown as mean \pm SEM. $N=6-12$. Figure S2. TF does not modify ILK expression in monocytes in vitro or in skeletal muscle in vitro and in vivo. A) Deprived cultured monocytes THP1 and B) myoblasts $\mathrm{C} 2 \mathrm{C} 12$ were treated with TF $50 \mu \mathrm{M}$ or vehicle (CT) $24 \mathrm{~h}$ and ILK mRNA expression fold changes were analyzed by RT-qPCR and normalized to $\beta$-actin. C Control mice (WT) were challenged to high fat diet (HFD) for 2 weeks and subjected to TF (50 microg/Kg/day, i.p.) or vehicle (VH). Afterward, animals were fasted overnight, weighed, sacrificed and vastus lateralis were dissected. Fold changes of ILK mRNA expression analyzed by RT-qPCR and normalized to $\beta$-actin. Data are shown as mean \pm SEM. $N=6-12$.

\section{Acknowledgements}

F-actin confocal images were determined by the Confocal Microscopy Service (ICTS 'NANBIOSIS' U17) of the Biomedical Research Networking Center on Bioengineering, Biomaterials and Nanomedicine (CIBER-BBN) and pharmacokinetics were determined in the Applied Chemistry and Biotechnology Center (CQAB), both at Universidad de Alcalá. We thank Isabel Trabado and Dr. Leonor Nozal for helping us to perform the experiments in these Centers.

\section{Authors' contributions}

MRP and DRP directed equally the work. SDF, MRP, DRP conceived the study. SDF designed the experimental plan, performed experiments, and wrote the manuscript. MG and MHV performed experiments, analyzed, and interpreted the data. SC, EGC and DGA performed experiments. LC and MP revised critically the intellectual content of the project. All authors approved the final submitted version. All authors read and approved the final manuscript.

\section{Funding}

This work was supported by co-founded grants from Instituto de Salud Carlos III (ISCIII), Comunidad de Madrid (NovelRen) and FEDER funds [Grants PI14/01939, PI14/02075, PI17/01513, PI17/00625, PI20/00634, S2017/BMD-3751], the FEDER and ISCIII RETIC REDinREN programs [Grants RD12/0021/0006 and RD16/0009/0018].

\section{Availability of data and materials}

All data generated or analyzed during this study are included in this published article and its additional files.

\section{Declarations}

\section{Ethics approval and consent to participate}

This study does not involve human participants, human data or human tissue. Animal experiments have been approved by the Institutional Animal Care and Use Committees from Universidad de Alcalá and Comunidad de Madrid (PROEX 230/16), in agreement with the guidelines established by the European Community Council Directives (2010/63/EU).

\section{Consent for publication}

Not applicable.

\section{Competing interests}

The authors declare that they have no competing interests.

\section{Author details}

${ }^{1}$ Departamento de Biología de Sistemas, Unidad Fisiología, Facultad de Medicina, Universidad de Alcalá, A2 KM 33,600, Campus, 28805 Alcalá de Henares (Madrid), Spain. ${ }^{2}$ Instituto Ramon Y Cajal de Investigación Sanitaria, Fundación Renal Iñigo Álvarez de Toledo and RedInRen from Instituto de Salud Carlos III, and NOVELREN from Comunidad de Madrid, Madrid, Spain. ${ }^{3}$ Graphenano Medical Care S.L., Madrid, Spain. ${ }^{4}$ Biomedical Research
Foundation from Hospital Universitario Príncipe de Asturias, Alcalá de Henares, Spain. ${ }^{5}$ Instituto de Investigación Sanitaria de Santiago, Servizo Galego de Saúde, A Coruña, Spain. ${ }^{6}$ Nephrology Unit from Hospital Príncipe de Asturias and Department of Medicine from Universidad de Alcalá, Alcalá de Henares, Spain.

Received: 7 September 2021 Accepted: 14 January 2022

Published online: 28 January 2022

\section{References}

1. O'Rourke RW. Adipose tissue and the physiologic underpinnings of metabolic disease. Surg Obes Relat Dis. 2018;14(11):1755-63. https://doi. org/10.1016/j.soard.2018.07.032.

2. Ali AT, Hochfeld WE, Myburgh R, Pepper MS. Adipocyte and adipogenesis. Eur J Cell Biol. 2013;92(6-7):229-36. https://doi.org/10.1016/j.ejcb.2013. 06.001.

3. Storch J, Thumser AE. The fatty acid transport function of fatty acidbinding proteins. Biochim Biophys Acta. 2000;1486:28-44. https://doi. org/10.1016/S1388-1981(00)00046-9.

4. Schweiger M, Schreiber R, Haemmerle G, Lass A, Fledelius C, Jacobsen P, Tornqvist $H$, Zechner R, Zimmermann R. Adipose triglyceride lipase and hormone-sensitive lipase are the major enzymes in adipose tissue triacylglycerol catabolism. J Biol Chem. 2006;281(52):40236-41. https://doi.org/ 10.1074/jbc.M608048200.

5. Pope BD, Warren CR, Parker KK, Cowan CA. Microenvironmental control of adipocyte fate and function. Trends Cell Biol. 2016;26(10):745-55. https:// doi.org/10.1016/j.tcb.2016.05.005.

6. Park A, Kim WK, Bae KH. Distinction of white, beige and brown adipocytes derived from mesenchymal stem cells. World J Stem Cells. 2014;6(1):3342. https://doi.org/10.4252/wjsc.v6.i1.33.

7. Zhang T, Qin X, Cao Y, Zhang J, Zhao J. Sea buckthorn (Hippophae rhamnoides L.) oil enhances proliferation, adipocytes differentiation and insulin sensitivity in 3T3-L1 cells. Food Sci Biotechnol. 2020;29(11):1511-8. https://doi.org/10.1007/s10068-020-00817-4.

8. Harms M, Seale P. Brown and beige fat: development, function and therapeutic potential. Nat Med. 2013;19(10):1252-63. https://doi.org/10.1038/ $\mathrm{nm} .3361$.

9. Tharp KM, Stahl A. Bioengineering beige adipose tissue therapeutics. Front Endocrinol (Lausanne). 2015;20(6):164. https://doi.org/10.3389/ fendo.2015.00164.61.

10. Shabalina IG, Petrovic N, de Jong JM, Kalinovich AV, Cannon B, Nedergaard J. UCP1 in brite/beige adipose tissue mitochondria is functionally thermogenic. Cell Rep. 2013;5(5):1196-203. https://doi.org/10.1016/j. celrep.2013.10.044.

11. Hoffmann JM, Grünberg JR, Church C, Elias I, Palsdottir V, Jansson JO, Bosch F, Hammarstedt A, Hedjazifar S, Smith U. BMP4 gene therapy in mature mice reduces BAT activation but protects from obesity by browning subcutaneous adipose tissue. Cell Rep. 2017;20(5):1038-49. https:// doi.org/10.1016/j.celrep.2017.07.020.

12. Kazak L, Chouchani ET, Jedrychowski MP, Erickson BK, Shinoda K, Cohen P, Vetrivelan R, Lu GZ, Laznik-Bogoslavski D, Hasenfuss SC, Kajimura S, Gygi $\mathrm{SP}$, Spiegelman BM. A creatine-driven substrate cycle enhances energy expenditure and thermogenesis in beige fat. Cell. 2015;163(3):643-55. https://doi.org/10.1016/j.cell.2015.09.035.

13. Rodriguez A, Catalan V, Gomez-Ambrosi J, Fruhbeck G. Aquaglyceroporins serve as metabolic gateways in adiposity and insulin resistance control. Cell Cycle. 2011;10:1548-56.

14. da Silva IV, Díaz-Sáez F, Zorzano A, Gumà A, Camps M, Soveral G. Aquaglyceroporins are differentially expressed in beige and white adipocytes. Int J Mol Sci. 2020;21(2):610. https://doi.org/10.3390/ijms21020610.

15. Gahmberg CG, Fagerholm SC, Nurmi SM, Chavakis T, Marchesan S, Grönholm M. Regulation of integrin activity and signalling. Biochim Biophys Acta. 2009;1790(6):431-44. https://doi.org/10.1016/j.bbagen.2009.03.007.

16. Féral CC, Neels JG, Kummer C, Slepak M, Olefsky JM, Ginsberg MH. Blockade of alpha4 integrin signaling ameliorates the metabolic consequences of high-fat diet-induced obesity. Diabetes. 2008;57(7):1842-51. https:// doi.org/10.2337/db07-1751.

17. Meakin PJ, Morrison VL, Sneddon CC, Savinko T, Uotila L, Jalicy SM, Gabriel $J$, Kang L, Ashford ML, Fagerholm SC. Mice lacking beta2-integrin 
function remain glucose tolerant in spite of insulin resistance, neutrophil infiltration and inflammation. PLOS ONE. 2015;10(9): e0138872. https:// doi.org/10.1371/journal.pone.0138872.

18. Bouvard D, Brakebusch C, Gustafsson E, Aszódi A, Bengtsson T, Berna A, Fässler R. Functional consequences of integrin gene mutations in mice. Circ Res. 2001;89(3):211-23. https://doi.org/10.1161/hh1501.094874.

19. Ruiz-Ojeda FJ, Wang J, Bäcker T, Krueger M, Zamani S, Rosowski S, Gruber T, Onogi Y, Feuchtinger A, Schulz TJ, Fässler R, Müller TD, García-Cáceres C, Meier M, Blüher M, Ussar S. Active integrins regulate white adipose tissue insulin sensitivity and brown fat thermogenesis. Mol Metab. 2021;7(45): 101147. https://doi.org/10.1016/j.molmet.2020.101147.

20. Liu J, DeYoung SM, Zhang M, Zhang M, Cheng A, Saltiel AR. Changes in integrin expression during adipocyte differentiation. Cell Metab. 2005;2(3):165-77. https://doi.org/10.1016/j.cmet.2005.08.006.

21. Luo S, Shi Q, Li W, Wu W, Zha Z. ITGB1 promotes the chondrogenic differentiation of human adipose-derived mesenchymal stem cells by activating the ERK signaling. J Mol Histol. 2020;51(6):729-39. https://doi. org/10.1007/s10735-020-09918-0.

22. Nilsson S, Kaniowska D, Brakebusch C, Fässler R, Johansson S. Threonine 788 in integrin subunit beta1 regulates integrin activation. Exp Cell Res. 2006;312(6):844-53. https://doi.org/10.1016/j.yexcr.2005.12.001.

23. Armani A, Mammi C, Marzolla V, Calanchini M, Antelmi A, Rosano GM, Fabbri A, Caprio M. Cellular models for understanding adipogenesis, adipose dysfunction, and obesity. J Cell Biochem. 2010;110(3):564-72. https://doi.org/10.1002/jcb.22598.

24. Liu F, He J, Wang H, Zhu D, Bi Y. Adipose morphology: a critical factor in regulation of human metabolic diseases and adipose tissue dysfunction. Obes Surg. 2020;30(12):5086-100. https://doi.org/10.1007/s11695-02004983-6 (Epub 2020 Oct 6)

25. Hansson B, Morén B, Fryklund C, Vliex L, Wasserstrom S, Albinsson S, Berger K, Stenkula KG. Adipose cell size changes are associated with a drastic actin remodeling. Sci Rep. 2019;9(1):12941. https://doi.org/10. 1038/s41598-019-49418-0.

26. Chen L, Hu H, Qiu W, Shi K, Kassem M. Actin depolymerization enhances adipogenic differentiation in human stromal stem cells. Stem Cell Res. 2018:29:76-83. https://doi.org/10.1016/.scr.2018.03.010.

27. Kim JI, Park J, Ji Y, Jo K, Han SM, Sohn JH, Shin KC, Han JS, Jeon YG, Nahmgoong H, Han KH, Kim J, Kim S, Choe SS, Kim JB. During adipocyte remodeling, lipid droplet configurations regulate insulin sensitivity through F-actin and G-actin reorganization. Mol Cell Biol. 2019;39(20):e00210-e219. https://doi.org/10.1128/MCB.00210-19.

28. Zhong XJ, Shen XD, Wen JB, Kong Y, Chu JJ, Yan GQ, LiT, Liu D, Wu MQ, Zeng GH, He M, Huang QR. Osteopontin-induced brown adipogenesis from white preadipocytes through a PI3K-AKT dependent signaling. Biochem Biophys Res Commun. 2015;459(3):553-9. https://doi.org/10. 1016/j.bbrc.2015.02.153

29. Souza-Moreira L, Soares VC, Dias SDSG, Bozza PT. Adipose-derived mesenchymal stromal cells modulate lipid metabolism and lipid droplet biogenesis via AKT/mTOR -PPARY signalling in macrophages. Sci Rep. 2019;9(1):20304. https://doi.org/10.1038/s41598-019-56835-8.

30. Kim AR, Yoon BK, Park H, Seok JW, Choi H, Yu JH, Choi Y, Song SJ, Kim A, Kim JW. Caffeine inhibits adipogenesis through modulation of mitotic clonal expansion and the AKT/GSK3 pathway in 3T3-L1 adipocytes. BMB Rep. 2016;49(2):111-5. https://doi.org/10.5483/bmbrep.2016.49.2.128.

31. Xu B, Ju Y, Song G. Role of p38, ERK1/2, focal adhesion kinase, RhoA/ROCK and cytoskeleton in the adipogenesis of human mesenchymal stem cells. J Biosci Bioeng. 2014;117(5):624-31. https://doi.org/10.1016/j.jbiosc.2013. 10.018 .

32. Boulter E, Van Obberghen-Schilling E. Integrin-linked kinase and its partners: a modular platform regulating cell-matrix adhesion dynamics and cytoskeletal organization. Eur J Cell Biol. 2006;85(3-4):255-63. https://doi. org/10.1016/j.ejcb.2005.09.002.

33. Tseng C, Kolonin MG. Proteolytic isoforms of SPARC induce adipose stromal cell mobilization in obesity. Stem Cells. 2016;34(1):174-90. https:// doi.org/10.1002/stem.2192.

34. Loibl M, Lang S, Hanke A, Herrmann M, Huber M, Brockhoff G, Klein S, Nerlich M, Angele P, Prantl L, Gehmert S. Leukocyte-reduced platelet-rich plasma alters protein expression of adipose tissue-derived mesenchymal stem cells. Plast Reconstr Surg. 2016;138(2):397-408. https://doi.org/10. 1097/PRS.0000000000002388.
35. Goessler UR, Bugert P, Bieback K, Stern-Straeter J, Bran G, Hörmann $\mathrm{K}$, Riedel F. Integrin expression in stem cells from bone marrow and adipose tissue during chondrogenic differentiation. Int J Mol Med. 2008:21(3):271-9

36. Hatem-Vaquero M, Griera M, Garcia-Ayuso D, Campillo S, Bohorquez L, Calleros L, Rodriguez-Puyol D, Rodriguez-Puyol M, de Frutos S. Integrin linked kinase (ILK) downregulation as an early event during the development of metabolic alterations in a short-term high fat diet mice model. Cell Physiol Biochem. 2020;54(1):71-87. https://doi.org/10.33594/00000 0206.

37. Hatem-Vaquero M, Griera M, García-Jerez A, Luengo A, Álvarez J, Rubio JA Calleros L, Rodríguez-Puyol D, Rodríguez-Puyol M, De Frutos S. Peripheral insulin resistance in ILK-depleted mice by reduction of GLUT4 expression. J Endocrinol. 2017;234(2):115-28.

38. Bugler-Lamb AR, Hasib A, Weng X, Hennayake CK, Lin C, McCrimmon RJ, Stimson RH, Ashford MLJ, Wasserman DH, Kang L. Adipocyte integrinlinked kinase plays a key role in the development of diet-induced adipose insulin resistance in male mice. Mol Metab. 2021;26(49): 101197. https://doi.org/10.1016/j.molmet.2021.101197.

39. Kapp TG, Rechenmacher F, Neubauer S, Maltsev OV, Cavalcanti-Adam EA, Zarka R, Reuning U, Notni J, Wester HJ, Mas-Moruno C, Spatz J, Geiger $\mathrm{B}$, Kessler H. A comprehensive evaluation of the activity and selectivity profile of ligands for RGD-binding integrins. Sci Rep. 2017;11(7):39805. https://doi.org/10.1038/srep39805.

40. King S, Short M, Harmon C. Glycoprotein Ilb/llla inhibitors: the resurgence of tirofiban. Vascul Pharmacol. 2016;78:10-6. https://doi.org/10.1016/j. vph.2015.07.008.

41. Cully M. Integrin-targeted therapies branch out. Nat Rev Drug Discov 2020;19(11):739-41. https://doi.org/10.1038/d41573-020-00180-3.

42. Giordano A, Romano S, D'Angelillo A, Corcione N, Messina S, Avellino R, Biondi-Zoccai G, Ferraro P, Romano MF. Tirofiban counteracts endothelial cell apoptosis through the VEGF/NEGFR2/pAkt axis. Vascul Pharmacol. 2016:80:67-74. https://doi.org/10.1016/j.vph.2015.12.001.

43. Giordano A, Romano S, Corcione N, Frati G, Zoccai GB, Ferraro P, Messina $S$, Ottolini S, Romano MF. Tirofiban positively regulates $\beta 1$ Integrin and favours endothelial cell growth on polylactic acid biopolymer vascular scaffold (BVS). J Cardiovasc Transl Res. 2018;11(3):201-9. https://doi.org/ 10.1007/s12265-018-9805-1.

44. Gao HQ, Xu SD, Li JR, Zheng J, Sun LZ. Tirofiban promotes the proliferation of human umbilical vein endothelial cells in vitro via enhanced vascular endothelial growth factor expression. Transplant Proc. 2020:52(1):419-22. https://doi.org/10.1016/.transproceed.2019.10.007.

45. Heinzmann ACA, Karel MFA, Coenen DM, Vajen T, Meulendijks NMM, Nagy M, Suylen DPL, Cosemans JMEM, Heemskerk JWM, Hackeng TM, Koenen RR. Complementary roles of platelet allb $\beta 3$ integrin, phosphatidylserine exposure and cytoskeletal rearrangement in the release of extracellular vesicles. Atherosclerosis. 2020;310:17-25. https://doi.org/10. 1016/j.atherosclerosis.2020.07.015.

46. Schmitt-Sody M, Metz P, Gottschalk O, Zysk S, Birkenmaier C, Goebl M, von Schulze PC, Veihelmann A, Jansson V. Selective inhibition of platelets by the GPIIb/IIla receptor antagonist Tirofiban reduces leukocyteendothelial cell interaction in murine antigen-induced arthritis. Inflamm Res. 2007:56(10):414-20. https://doi.org/10.1007/s00011-007-7024-3.

47. Jiang L, Song XH, Liu P, Zeng CL, Huang ZS, Zhu LJ, Jiang YZ, Ouyang HW, $\mathrm{Hu}$ H. Platelet-mediated mesenchymal stem cells homing to the lung reduces monocrotaline-induced rat pulmonary hypertension. Cell Transplant. 2012;21(7):1463-75. https://doi.org/10.3727/096368912X640529.

48. Guan W, Wang Z, Liu Y, Han Y, Ren H, Eric Wang W, Yang J, Zhou L, Zeng C. Protective effects of tirofiban on ischemia/reperfusion-induced renal injury in vivo and in vitro. Eur J Pharmacol. 2015;15(761):144-52. https:// doi.org/10.1016/j.ejphar.2015.05.009.

49. Chamorro-Jorganes A, Calleros L, Griera M, Saura M, Luengo A, RodriguezPuyol D, Rodriguez-Puyol M. Fibronectin upregulates CGMP-dependent protein kinase type I $\beta$ through C/EBP transcription factor activation in contractile cells. Am J Physiol Cell Physiol. 2011;300(3):C683-91. https:// doi.org/10.1152/ajpcell.00251.2010.

50. Ruiz-Torres MP, Griera M, Chamorro A, Díez-Marqués ML, Rodríguez-Puyol $D$, Rodríguez-Puyol M. Tirofiban increases soluble guanylate cyclase in rat vascular walls: pharmacological and pathophysiological consequences. Cardiovasc Res. 2009;82(1):125-32. https://doi.org/10.1093/cvr/cvn359. 
51. Tang QQ, Otto TC, Lane MD. Commitment of $\mathrm{C} 3 \mathrm{H} 10 \mathrm{~T} 1 / 2$ pluripotent stem cells to the adipocyte lineage. Proc Natl Acad Sci U S A. 2004;101(26):9607-11. https://doi.org/10.1073/pnas.0403100101.

52. Lee MJ, Pickering RT, Puri V. Prolonged efficiency of siRNA-mediated gene silencing in primary cultures of human preadipocytes and adipocytes. Obesity (Silver Spring, Md). 2014;22:1064-9. https://doi.org/10.1002/oby. 20641.

53. Isidor MS, Winther S, Basse AL, Petersen MC, Cannon B, Nedergaard J, Hansen JB. An siRNA-based method for efficient silencing of gene expression in mature brown adipocytes. Adipocyte. 2015;5(2):175-85. https:// doi.org/10.1080/21623945.2015.1111972.

54. Peuhu E, Salomaa SI, De Franceschi N, Potter CS, Sundberg JP, Pouwels J. Integrin beta 1 inhibition alleviates the chronic hyperproliferative dermatitis phenotype of SHARPIN-deficient mice. PLOS ONE. 2017;12(10): e0186628. https://doi.org/10.1371/journal.pone.0186628.

55. Hyväri L, Ojansivu M, Juntunen M, Kartasalo K, Miettinen S, Vanhatupa S Focal adhesion kinase and ROCK signaling are switch-like regulators of human adipose stem cell differentiation towards osteogenic and adipogenic lineages. Stem Cells Int. 2018;12(2018):2190657. https://doi.org/10. 1155/2018/2190657.

56. Vickers S, Theoharides AD, Arison B, Balani SK, Cui D, Duncan CA, Ellis JD, Gorham LM, Polsky SL, Prueksaritanont T, Ramjit HG, Slaughter DE, Vyas KP. In vitro and in vivo studies on the metabolism of tirofiban. Drug Metab Dispos. 1999;27(11):1360-6.

57. Rubin J, Sen B. Actin up in the nucleus: regulation of actin structures modulates mesenchymal stem cell differentiation. Trans Am Clin Climatol Assoc. 2017;128:180-92.

58. Spiegelman BM. Farmer SR decreases in tubulin and actin gene expression prior to morphological differentiation of $3 T 3$ adipocytes. Cell. 1982;29(1):53-60.

59. Müller P, Langenbach A, Kaminski A, Rychly J. Modulating the actin cytoskeleton affects mechanically induced signal transduction and differentiation in mesenchymal stem cells. PLoS ONE. 2013;8(7): e71283. https://doi.org/10.1371/journal.pone.0071283.

60. Feng T, Szabo E, Dziak E, Opas M. Cytoskeletal disassembly and cell rounding promotes adipogenesis from ES cells. Stem Cell Rev Rep. 2010;6(1):74-85. https://doi.org/10.1007/s12015-010-9115-8.

61. Mathieu PS, Loboa EG. Cytoskeletal and focal adhesion influences on mesenchymal stem cell shape, mechanical properties, and differentiation down osteogenic, adipogenic, and chondrogenic pathways. Tissue Eng Part B Rev. 2012;18(6):436-44. https://doi.org/10.1089/ten.TEB.2012.0014.

62. Janota CS, Calero-Cuenca FJ, Gomes ER. The role of the cell nucleus in mechanotransduction. Curr Opin Cell Biol. 2020;63:204-11. https://doi. org/10.1016/j.ceb.2020.03.001.

63. Murakami K, Eguchi J, Hida K, Nakatsuka A, Katayama A, Sakurai M, Choshi H, Furutani M, Ogawa D, Takei K, Otsuka F, Wada J. Antiobesity action of ACAM by modulating the dynamics of cell adhesion and actin polymerization in adipocytes. Diabetes. 2016;65(5):1255-67. https://doi.org/10. 2337/db15-1304.

\section{Publisher's Note}

Springer Nature remains neutral with regard to jurisdictional claims in published maps and institutional affiliations.

Ready to submit your research? Choose BMC and benefit from:

- fast, convenient online submission

- thorough peer review by experienced researchers in your field

- rapid publication on acceptance

- support for research data, including large and complex data types

- gold Open Access which fosters wider collaboration and increased citations

- maximum visibility for your research: over $100 \mathrm{M}$ website views per year

At BMC, research is always in progress.

Learn more biomedcentral.com/submissions 